\title{
Le rôle des traces dans le système immunitaire : des anticorps au corps
}

Véronique THOMAS-VASLIN ${ }^{1}$

\section{CONTEXTUALISATION DU PROPOS}

\section{Le cadre général : les systèmes complexes multi-échelles}

Ce chapitre va permettre d'illustrer le concept de «traces » dans le corps humain, générées par le système immunitaire et transmises entre les générations. Conformément à la perspective du E. Laboratoire HumanTrace Complex System ${ }^{2}$, qui constitue le cadre institutionnel de ce travail, nous nous placerons dans des systèmes multi-échelles. Nous passerons alternativement de l'échelle du corps (notre ressenti et ce qui est visuel) à l'échelle invisible des anticorps et autres immuno-récepteurs capables d'identifier les molécules de notre corps et de notre entourage, au cours du temps, lors de traces processuelles. La première partie porte de façon générale sur les traces biologiques qui peuvent être recensées par l'homme, le chercheur en immunologie, le médecin... puis elle brosse quelques aspects généraux du comportement du système immunitaire. La notion de signe-trace, présentée par B. Galinon-Mélénec dans le tome 1 de L'Homme-trace $(2011)^{3}$ est utilisée fréquemment dans ce chapitre sans pour autant que le paradigme du même nom ${ }^{4}$ soit repris dans sa globalité. Quelques figures illustrent les propos et il est conseillé d'y revenir au cours de la lecture pour faciliter le cheminement entre les échelles, dans le temps et l'espace dans ce système complexe qu'est le système immunitaire. Comprendre la complexité du système immunitaire est un défi du réseau interdisciplinaire ImmunoComplexiT ${ }^{5}$ (Thomas-Vaslin 2015a). Nous proposons en dernière instance de revisiter les notions de frontière du soi, de perception du corps, du ressenti de l'invisible pour l'inspection du monde, abordées par les philosophes, ce qui nous amène à interroger l'émersiologie telle que la définit B. Andrieu (Andrieu 2016). Nous espérons que les niveaux de précision sur les différents aspects permettront à des non-spécialistes de découvrir ou mieux appréhender le rôle primordial du système immunitaire dans la trace du vivant et du corps et les conséquences au niveau du macrocosme sociétal ${ }^{6}$.

\section{Signes-traces et Homme-trace dans notre perspective}

Les recherches contemporaines sur le vivant démontrent que l'organisation, la reproduction, la diversité, la viabilité et la résilience des systèmes vivants sont interconnectés à différents niveaux biologiques, sous forme de micro et macro écosystèmes. Aussi, au-delà des particularités spécifiques, les systèmes

\footnotetext{
1 Sorbonne Universités, UPMC Univ Paris 06, INSERM, CNRS, Immunologie-ImmunopathologieImmunothérapeutique(I3) ; Paris.

${ }^{2} \mathrm{http} / / /$ tinyurl.com/human-trace-unesco.

${ }^{3}$ Galinon-Mélénec B., « Fragments théoriques du signe-trace, propos sur le corps communicant », in L'Homme-trace, Perspectives anthropologiques des traces contemporaines, Paris, CNRS Éditions, série L'Homme-trace, tome 1, 2011, p. 191-213.

${ }^{4}$ Le paradigme des signes-traces pris dans son ensemble imposerait une dimension épistémologique de l'interprétation, c'est-à-dire la prise en compte des signes-traces cognitifs de l'interprétant dans son analyse de l'objet (ici, le signe-trace biologique). Cf. Galinon-Mélénec B., « Expérience incarnée, construction cognitive et jugement : Le rôle des « signestraces » du corps dans la signification », dans Boutaud J.J., Berthelot-Guiet K. (dir.), "Revue Française des Sciences de l'Information et de la Communication », 2013. En ligne sur http://rfsic.revues.org/487

${ }^{5}$ http://www.immunocomplexit.net/.

${ }^{6}$ Pour les lecteurs qui souhaitent approfondir, un texte du même auteur est dédié à la complexité multi-échelle du système immunitaire : Évolution, du chaos aux fractales, en s'appuyant sur des aspects de modélisation dynamique qui « retrace » ce que l'on en comprend (Thomas-Vaslin 2015b).
} 
biologiques complexes partagent-ils tous une certaine historicité. Ce qui signifie que tout organisme vivant porte en lui les signes-traces de cette histoire commune et individuelle (Galinon-Mélénec, 2011) ${ }^{7}$.

Si l'on se centre sur l'Homme, il est acquis que la mise en place du système immunitaire commence dès la vie intra-utérine, alors même que le fœtus est protégé contre les microbes par sa mère. La cognition par le système immunitaire, des «antigènes » du fœtus et de ceux de la mère contribue à la construction de l'identité moléculaire de l'individu, en particulier via les «anticorps ${ }^{8}$. Dès la naissance, ce système immunitaire est confronté potentiellement à tous les «antigènes " ${ }^{9}$ et à tous les microbes de l'environnement et il se rappellera tout au long de la vie de cette rencontre... ce qui lui permettra de s'adapter et de répondre avec plus d'efficacité à une nouvelle confrontation. Ainsi, jour après jour, le système immunitaire est confronté à notre « microbiote ${ }^{10}$ indispensable à notre vie, qui compose environ $50 \%$ des cellules de notre organisme et $90 \%$ de l'ADN. Le système immunitaire garde une trace de l'histoire moléculaire et en particulier infectieuse de l'organisme par la mémoire immunologique.

L'évolution physiologique d'un organisme continue au cours de la vie. Les traces de ce parcours de vie semé d'embûches et de perturbations liées aux contraintes de l'environnement - orientent la trajectoire des composants moléculaires, des cellules, leur survie ou mort cellulaire et jusqu'aux grandes fonctions de l'organisme. Selon les traces laissées par le chemin parcouru, les systèmes intracorporels et le corps dans sa globalité sont plus ou moins robustes et résilients aux perturbations et au vieillissement.

Les récentes technologies qui génèrent de nouvelles entités (les produits chimiques, les allergènes, les OGM...) ou de nouveaux procédés (l'hygiène, la désinfection, la vaccination, la transformation des aliments, les thérapies, les naissances par césarienne...) ne sont pas neutres dans cette évolution. Elles impactent l'évolution du corps en modifiant la réactivité du système immunitaire.

Notre propos se situe donc bien dans un des aspects de la définition de l' «Homme-trace », celui qui insiste sur le fait que le corps de l'humain se construit et se développe en agrégeant des traces.

Aujourd'hui, diverses techniques d'observation portant sur l'organisation multi-échelle des systèmes, de la molécule à l'organisme, permettent de détecter chez l'Homme des combinaisons de bio-marqueurs, formes de signes-traces $d u$ corps qui permettent de distinguer tel ou tel comportement pathologique. Dans la mesure où détecter ces signes-traces ${ }^{11}$ permet de mettre en œuvre des thérapies qui améliorent la longévité, on comprend que la question dont nous traiterons ici pour illustrer le paradigme de l'Homme-trace constitue un intérêt majeur de ce $\mathrm{XXI}^{\mathrm{e}}$ siècle.

\section{Les traces des systèmes vivants}

Les systèmes vivants multi-échelles, de la cellule à l'écosystème, évoluent dans l'espace et le temps selon une dynamique fonctionnelle de construction et déconstruction, liées à l'utilisation et la dissipation de l'énergie. Les contraintes et restrictions de l'environnement et les perturbations du système façonnent leur évolution et laisse des traces dans le "système" et "l'environnement" (si on considère une frontière virtuelle qui facilite notre compréhension mais qui sera discutée). L'ensemble de ces systèmes et sous-systèmes, reproducteurs et dissipatifs d'énergie quelle qu'en soit l'échelle, sont dépendants d'un métabolisme, de réparations et réplications, reçoivent des « input » et produisent des « output » (Rosen, 1958), processus si

\footnotetext{
${ }^{7}$ Galinon-Mélénec B., "The ICHNOS-ANTHROPOS or the foundations of a humanism of the trace”, in Parrend P., Bourgine P., Collet P. (eds.), From fields to territories to the planet, First Complex Systems Digital Campus World E-Conference 2015, Springer 2017. DOI : 10.1007/978-3-319-45901-1. Cf. From "Traces" and "Human Trace" to "Human-Trace Paradigm" - Springer, link.springer.com

${ }^{8}$ Le préfixe « anti » est tiré de la préposition grecque anti, exprimant notamment l'opposition et la protection contre un mal. Il peut aussi être interprété à partir du latin dans le sens de « en face de ». Le terme " antibiotique » par exemple désigne une substance produite par des micro-organismes et qui s'oppose à d'autres micro-organismes pathogènes. (Source : Le dictionnaire historique de le langue française, Alain Rey dir., 2006).

Anticorps : Molécule, immunoglobuline du système immunitaire d'abord décrite par Erhlich P. (prix Nobel en 1908) comme un récepteur spécifique préformé à la surface d'une cellule, ayant une activité anti-toxine. Puis par extension désigne les immunoglobulines portées et secrétées par les lymphocytes B qui sont capables de se complexer aux antigènes.

${ }^{9}$ Antigène : toute molécule reconnue par le système immunitaire et qui sélectionne des anticorps et provoque leur fabrication.

${ }^{10}$ Microbiote : ensemble des micro-organismes qui vivent dans des zones spécifiques de l'organisme, et désignant par exemple la flore l'intestinale, cutanée ou vaginale.

${ }^{11}$ Comme l'a expliqué B. Galinon-Mélénec dans la Préface du tome 3 de la série l'Homme-trace, le signe-trace émerge à partir d'interactions et sa perception et son interprétation sont limitées par plusieurs préalables dont « les processus d’identification, le présupposé interprétatif, l'implicite temporo-causal » (Galinon-Mélénec, 2015).
} 
spécifiques au vivant, qu'ils peuvent être considérés comme des traces-processuelles dynamiques ou évolutives.

On peut observer les «traces-statiques » («signes-traces» résultants de processus dynamique), patterns, empreintes évoquant les différentes espèces, les composants des organismes, du corps humain ou du système immunitaire, comme la résultante de sa composition observée à un moment. Ces «signes-traces » sont des messages pour le système vivant qui les perçoit, que ce soit un animal ou une cellule. La construction/déconstruction du corps participe à des traces dynamiques (nommées «traces-processuelles » dans la terminologie de Galinon-Mélénec) qui produisent "le corps" et ses changements au cours de l'évolution des espèces, de l'ontogénèse, de la différenciation et diversification des cellules (et en particulier des lymphocytes) et de l'organogénèse et du vieillissement. L'homme est ainsi un « construit de traces $\gg 12$. Il existe au cours de cette évolution du corps des combinaisons dynamiques de paramètres, objectivées par des signatures statiques (signes-traces) ou dynamiques (traces-processuelles) pour lesquelles l'organisme est viable et qui permettent une adaptabilité et une évolution du système ${ }^{13}$. Des perturbations du système entraînent des altérations du métabolisme, de la réparation et de la mémoire des cellules et des systèmes. Ces perturbations sont elles-mêmes des processus (traces-perturbations). Ces traces-processuelles spécifiques évoluent de l'ontogénèse au vieillissement de l'organisme.

\section{Observation de traces-immunologiques}

L'homme est producteur mais aussi observateur de traces, un «pisteur de traces » 14 à tous les niveaux d'échelle macroscopique et microscopique. Les «traces », selon l'acception commune du terme, autrement dit dans notre contexte, les signes-traces visibles ou les traces processuelles invisibles directement, peuvent être analysées par l'homme en tant que chercheur. Certains signes-traces peuvent être plus ou moins labiles et visibles pour l'observateur.

La mort est un signe-trace résultant de l'altération de processus indispensables à la vie des cellules et du corps. L'absence de cohérence de réparation et reproduction de nouvelles molécules ou cellules ou de sélection positive des lymphocytes, qui sont des processus importants pour le système immunitaire, entraîne la mort des cellules. La mort cause une décomposition dynamique (traces-processuelles) qui aboutit à une déstructuration du corps (la matière décomposée étant un signe-trace, le nécrobiome permet de mesurer la dynamique de décomposition). La décomposition post-mortem des corps a été initialement décrite par Hippocrate par la locution grecque «apoptose» ${ }^{15}$. À l'échelle microscopique cellulaire, le terme apoptose est maintenant utilisé pour décrire le processus de «mort cellulaire programmée » qui provoque la déstructuration ordonnée des cellules, sur laquelle nous reviendrons car elle fait partie des processus de sélection et signaux d'alerte du système immunitaire que nous allons traiter.

Les signatures biologiques sont des signes-traces, conséquences de processus complexes observés à moment donné et à différentes granularités, donc à des échelles grossières ou fines. Ces signatures peuvent être étudiées finement par le chercheur en immunologie et utilisées par le médecin pour le diagnostic médical, en particulier pour mieux appréhender le comportement du système immunitaire et ses conséquences pour notre corps. Les chercheurs peuvent s'aider d'anticorps spécifiques à des fins de détection de traces (signes-traces) de molécules biologiques ou chimiques (qui sont des antigènes). Les anticorps (souvent eux-mêmes couplés à des traceurs fluorescents permettant leur identification) révèlent la présence qualitative et quantitative d'antigènes en s'y fixant (trace-processuelle étudiée en laboratoire, selon des protocoles adéquats). Ils tracent ainsi au niveau cellulaire et moléculaire la progression de pathologies, la mémoire d'une infection, l'efficacité d'une vaccination, etc. Une sérologie avec la détection positive d'anticorps est le signe-trace par exemple d'une réponse immunitaire lors d'une primo-infection ou d'une vaccination. La biologie de systèmes s'appuie sur la recherche de bio-marqueurs - combinaisons de traceurs - pour définir les phénotypes des cellules immunitaires lors de leur différenciation et pour caractériser les molécules impliquées dans diverses réactions immunitaires ou pathologies.

La recherche de traceurs moléculaires ou cellulaires, pour la classification de pathologies complexes autoimmunes et inflammatoires, est actuellement développée pour le diagnostic mais aussi la thérapie ${ }^{16}$ et

\footnotetext{
${ }^{12}$ «L'Homme-trace est d'une part “construit de traces" et d'autre part un "producteur de traces” dans un milieu qui en interagissant avec lui participe à sa construction » (Galinon-Mélénec, 2013).

${ }^{13}$ Même si le système n'est jamais optimal.

${ }^{14}$ Galinon-Mélénec B., tome 3, p. 22, note 39.

${ }^{15}$ Cette locution « chûte des feuilles » a ensuite été utilisée pour décrire la « chute des os » par le médecin Hippocrate le Grand.

${ }^{16}$ Le LabEx Transimmunom étudie le « l'immunome » et le « microbiome » pour identifier des signes-traces de pathologies immunitaires afin de les re-classifier selon des biomarqueurs, https://www.transimmunom.fr/en/Research.
} 
V.Thomas-Vaslin dans «Des Traces du corps au corps-trace», Série L'Homme-trace, tome IV version du 6/4/2017

l'optimisation des réponses vaccinales (Six, Bellier et al., 2012). Ainsi peut-on sur la base de « signatures » de transcrits de gènes prédire l'efficacité d'un vaccin dès six heures après une vaccination, alors que la réponse immune cellulaire demandera une dizaine de jours pour être quantifiable (Derian, Bellier et al., 2016). Des molécules «traceurs» tels des isotopes radioactifs ou plus couramment des combinaisons de fluorochromes sont également largement utilisées pour étudier la dynamique des lymphocytes. Les cellules sont directement marquées ou révélées par la trace d'anticorps (signes-traces spécifiques) qui les ont reconnues (Thomas-Vaslin, Six et al., 2013a). De telles méthodologies permettent de quantifier in vivo la présence et la prolifération de lymphocytes (Thomas-Vaslin, Altes et al., 2008, Vibert et Thomas-Vaslin 2017) ou la destruction de tumeurs par des lymphocytes (Gottrand, Courau et al., 2015) ou encore les conséquences d'une immunosuppression et les signes-traces du vieillissement sur le système immunitaire (Thomas-Vaslin, Six et al., 2012).

\section{Simulation de traces-immunologiques}

Le chercheur peut également modéliser de façon dynamique les processus biologiques et immunologiques afin de mieux les comprendre et réaliser des simulations du comportement individuel et collectif et interactif des cellules, des populations cellulaires et des tissus et étudier d'éventuelles prédictions de comportement. Au-delà des équations mathématiques, les diagrammes de transition et langages graphiques utilisés en informatique permettent de représenter cette dynamique et de modéliser le cheminement des traces-processuelles des cellules. Ainsi peut-on suivre expérimentalement et modéliser les tracesprocessuelles des cellules du système immunitaire, tels que les lymphocytes, qui participent à des processus de différenciation, d'interaction, de prolifération, de sélection (mort) et de migration (Thomas-Vaslin, Altes et al., 2008, McEwan, Bersini et al., 2011, Bersini, Klatzmann et al., 2012, Thomas-Vaslin, Six et al., 2013 b). On peut dès lors suivre in vitro la transition d'état des lymphocytes, dans le cycle cellulaire ou vers la mort cellulaire au cours d'une réponse immunitaire et modéliser ces processus (Loap, Pascalie et al., soumis).

Un exemple du comportement des lymphocytes, de leur traçabilité expérimentale et des simulations de la dynamique de la trace-processuelle est illustré par la Figure 1.

\section{Trace de la dynamique des lymphocytes T}

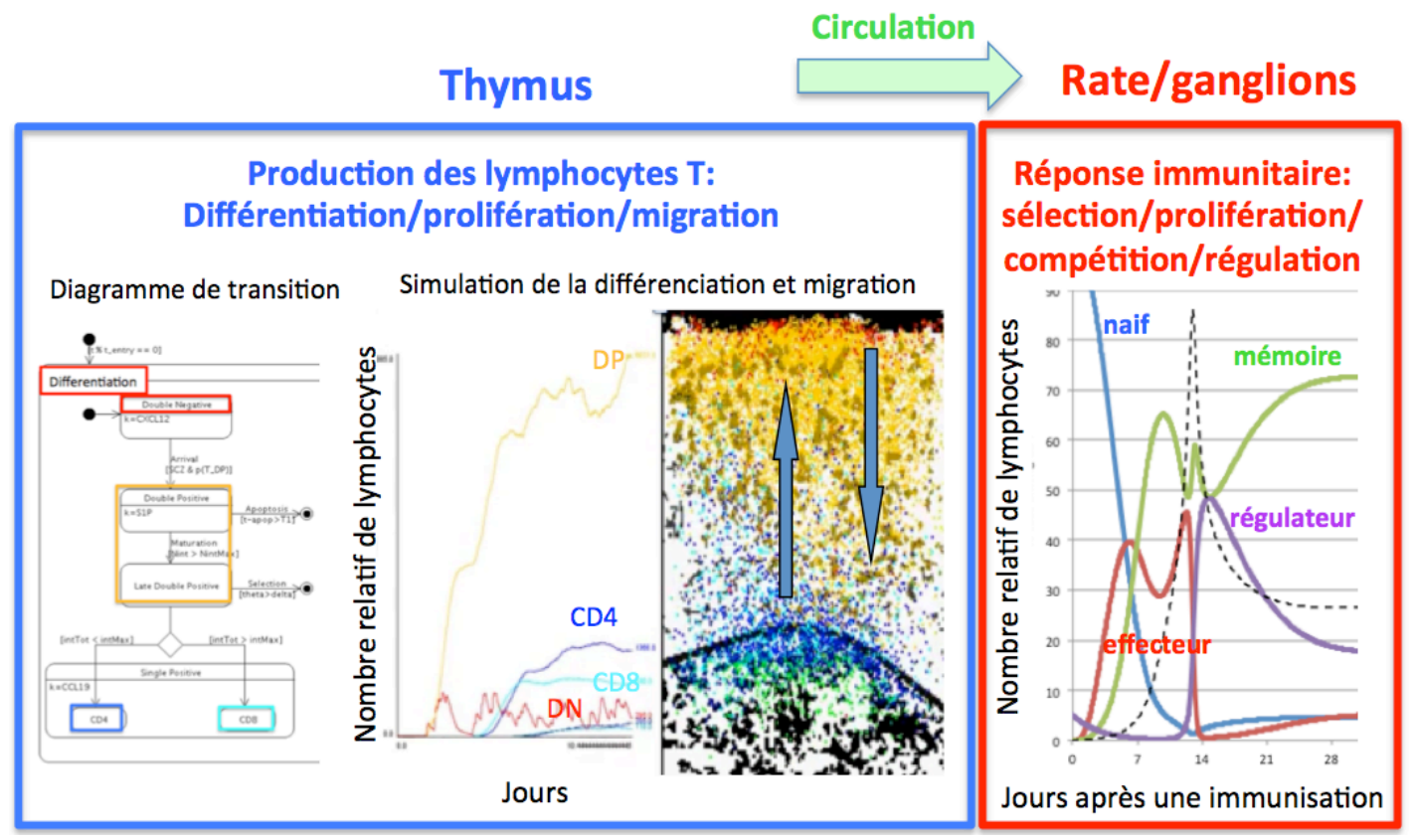

Figure 1.

Figure 1. Illustration de la trace produite et simulée par les lymphocytes dans le système immunitaire. La lignée de lymphocytes $T$ est produite dans le thymus (présenté dans le 
cadre bleu) par des étapes de différenciations séquentielles, représentées sur le diagramme de transition (exécutable par l'ordinateur). Les couleurs symbolisent la transition des cellules dans les différents stades de différenciation ( $D N->D P->C D 4$ ou CD8), pour aboutir à des lymphocytes T CD4 ou CD8 matures fonctionnels, analysées par des combinaisons de traceurs moléculaires. Les lymphocytes interagissent et migrent de façon orientée (flèches bleues) en suivant la trace de molécules chimiotactiques (chimiokines). Les thymocytes s'accumulent dans le thymus en formation jusqu'à un état $d$ '" homéo-dynamisme " permettant leur renouvellement permanent. Durant ces étapes les lymphocytes réarrangent certains gènes pour diversifier leur immuno-récepteur au niveau somatique, et former ainsi un récepteur protéique dont la séquence est spécifique à chaque lymphocyte. Ensuite, lors de processus de sélection massif, plus de $90 \%$ des lymphocytes meurent d'apoptose dans le thymus. Les thymocytes matures CD4 et CD8 qui échappent à la mort sortent alors du thymus et via la circulation entrent dans la rate ou les ganglions (cadre rouge) où ils participent aux réponses immunitaires. Lors d'une réponse immunitaire, certains lymphocytes "naïfs" (bleus) reconnaissent via leur immuno-récepteur spécifique le signal de l'antigène: ils sont sélectionnés, s'activent, prolifèrent et passent par différentes fonctions : ils deviennent lymphocytes " effecteurs" (rouge) puis "mémoire" (vert) (>90\% meurent), alors que d'autres lymphocytes "régulateurs" (violet) contrôlent la prolifération des lymphocytes effecteurs. Ainsi l'amplitude et la durée du pic de la réponse immunitaire (courbe pointillée) sont régulées pour normalement prévenir des réponses immunes excessives, auto-immunes ou inflammatoires (Thomas-Vaslin 2015b).

\section{Les Traces-Immunes : des traces processuelles au signe-trace et}

\section{leur visibilité}

Selon B. Galinon-Mélénec, "L’homme et le milieu entretiennent des relations permanentes. Le premier reçoit du second des flux (de matière, d'énergie, etc.) qui le modifient (directement ou indirectement) dans toute sa matière corps (y compris le cerveau). Le corps y répond, ce qui modifie le milieu en retour ${ }^{17}$ et «le concept d'extériorité » qui pose la relation dedans-dehors du corps sous forme de coupure est une métaphore spatiale. La frontière individu/environnement est visuelle. En fait, les corps sont poreux : le « dedans » porte en lui des « choses » venues du «dehors»; le « dehors » contient des « choses » en provenance de notre «dedans ». Si l'on maintient cette distinction utile bien que sujette à critique, ces « choses » peuvent être interprétées comme des traces d'interactions «individu/environnement ${ }^{18}$.

Il s'agit ici d'expliquer les traces de l'interaction entre le système immunitaire et son environnement moléculaire et cellulaire et l'intégration des écosystèmes qui entourent les individus. La nature de la trace en tant que "trace-processuelle » ou "signe-trace » qui en est issue et la transmission de diverses traces liées au système immunitaire, avec une visibilité à différentes échelles moléculaires, cellulaire, microscopique, macroscopiques, ou sociétales, sont exemplifiées dans le Tableau 1. Globalement, la traceimmune n'est pas statique mais constitue un ensemble dynamique de cellules et molécules en renouvellement permanent, dont on peut analyser la trace processuelle et les signes-traces émergents.

\footnotetext{
${ }^{17}$ Cf. Galinon-Mélénec B. «Épistémologie de la notion de trace », tome 3 de la série L'Homme-trace, p. 13.

${ }^{18}$ Ibid., p. 14.
} 


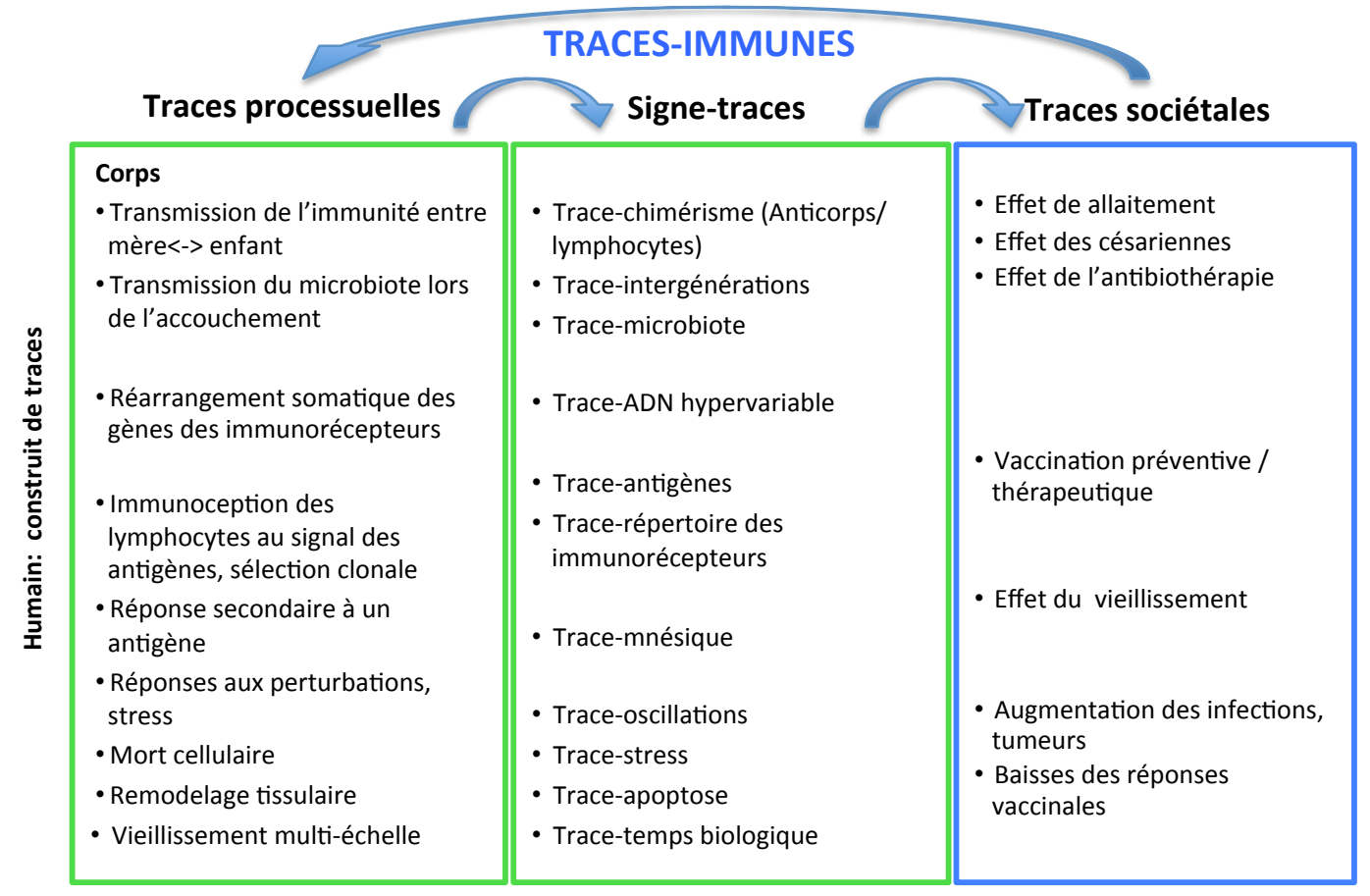

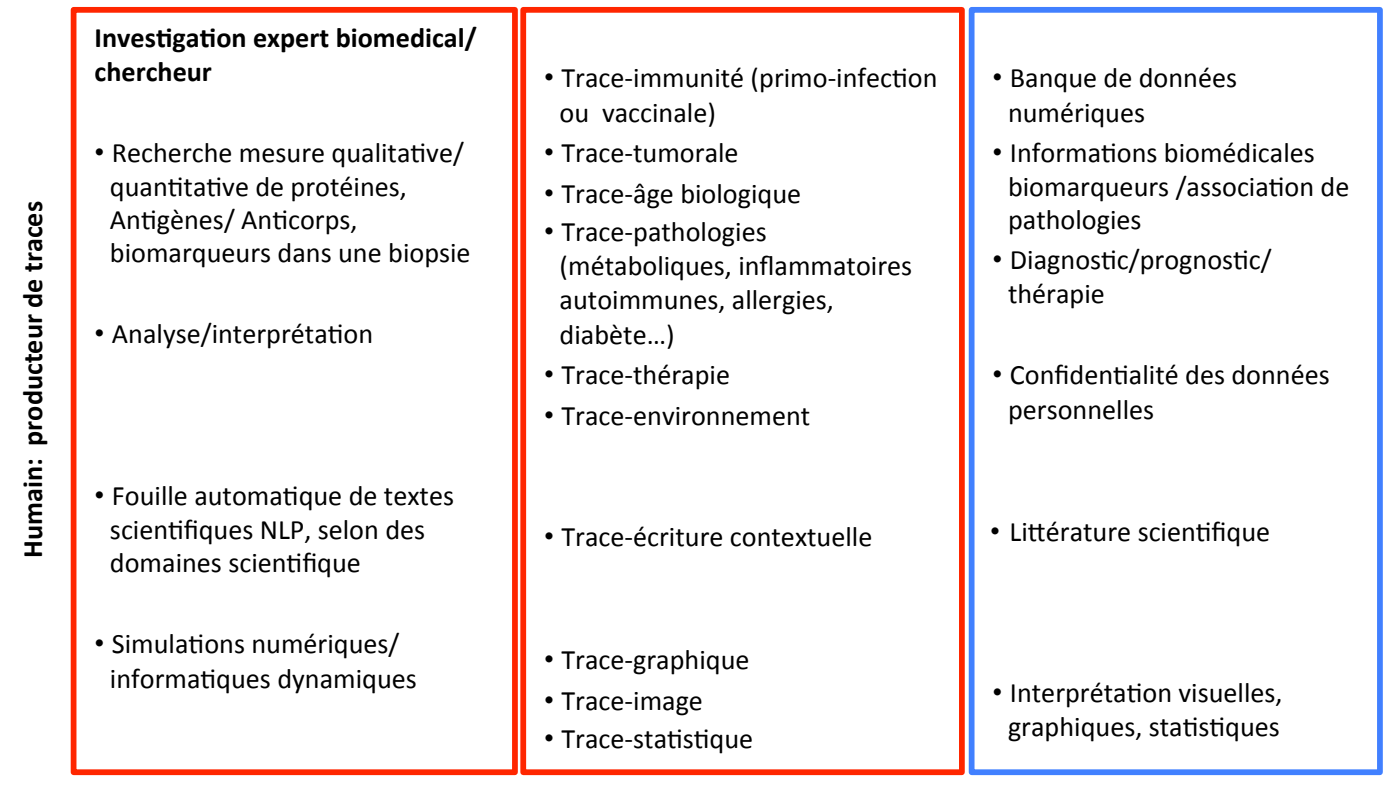

\section{Visibilité de la trace}

Tableau 1.

Les traces immunes sont multi-échelles. Ce sont des traces processuelles liées à la dynamique des espèces et du corps de l'homme qui est un produit de traces (issues du Darwinisme et Lamarckisme) qui reçoit des perturbations/contraintes de l'environnement et qui construit des traces et les transmet aux autres générations via son système immunitaire.

Il en résulte des signes-traces produits ou perçus par le système immunitaire qui évoluent, voire se dégradent avec l'âge, causant de nombreuses pathologies.

Au niveau macroscopique et sociétal émergent les effets des altérations corporelles et des technologies récentes qui peuvent être analysées ou produites par des experts chercheurs et dans le domaine biomédical, pour révéler ou modifier des traces perçues par le corps. 
Comme ces traces sont microscopiques elles ne sont donc pas nécessairement conscientes mais certaines sont révélées au niveau sociétal.

Il existe une récursivité des traces, car le comportement social peut influencer les traces processuelles immunes et en conséquence les signes-traces observés au niveau du système immunitaire ou des pathologies associées, dont on recherche l'identification par des signatures, des marqueurs biologiques.

Les experts en immunologie, les chercheurs, produisent des traces dans la littérature scientifique qui demandent en retour l'analyse de millions d'articles pour contextualiser la recherche et interpréter ces signes/indices observés dans des domaines particuliers (associant par exemple pathologies, anatomie, types cellulaires) ${ }^{19}$.

Les analyses et simulations numériques permettent d'interpréter et reproduire dans des modèles des processus qui opèrent au cours du temps et d'en montrer la trace par des graphes, des statistiques, des images, qui peuvent être recueillies et transmises dans des banques de données.

\section{COMPLEXITÉ DES TRACES DANS LE VIVANT, LE CORPS ET LE SYSTÈME IMMUNITAIRE}

\section{Évolution des écosystèmes multi-échelles : des espèces au système}

\section{immunitaire}

La biosphère et ses sous-systèmes, communauté, organismes, individus, cellules et molécules forment des écosystèmes complexes évolutifs donc qui produisent et transmettent des traces. Le système immunitaire est un de ces écosystèmes : il est formé par des populations de cellules diversifiées et interactives, telles que les lymphocytes, produits par le corps. De façon exceptionnelle et remarquable par rapport aux autres cellules du corps, les lymphocytes produisent durant leur différenciation une diversité somatique de molécules d'immuno-récepteurs diversifiés et sélectionnés, capables de communiquer avec les molécules de l'environnement. Nous allons tenter d'expliquer ces traces processuelles en progressant sur différentes échelles de temps et d'espace à l'aide d'exemples.

\section{Diversité et sélection naturelle : un processus multi-échelle}

Diversité et sélection naturelle sont des processus qui interviennent au cours de l'évolution des espèces, révélés par Darwin (Darwin, 1859). L'organisation et la connectivité des réseaux biologiques est progressive dans le temps et l'espace par cette diversification et sélection, ce qui crée des arborescences multi-échelles. La transmission de traces à diverses échelles a lieu au cours de l'évolution des espèces, de l'ontogénèse, lors de la différenciation des cellules, des tissus et des organes de l'organisme. Cette arborescence, de type fractal, est facilement visible au niveau du corps comme dans le réseau vasculaire ou les poumons. Cette arborescence se construit également lors de l'hématopoï̀se et du réarrangement somatique des gènes des immuno-récepteurs de chaque lymphocyte au niveau moléculaire pour produire une diversité de régions peptidiques hypervariables (Thomas-Vaslin, 2015b). La trace dynamique évolutive, connective et cognitive du système immunitaire est schématiquement représentée sur la Figure 2.

\footnotetext{
${ }^{19}$ La recherche dans la littérature est actuellement automatisée : Bedhiafi W., Thomas-Vaslin V., Benammar Elgaaied A., et Six A. (submitted), OntoContext, a new python package for gene contextualisation based on the annotation of biomedical texts.
} 


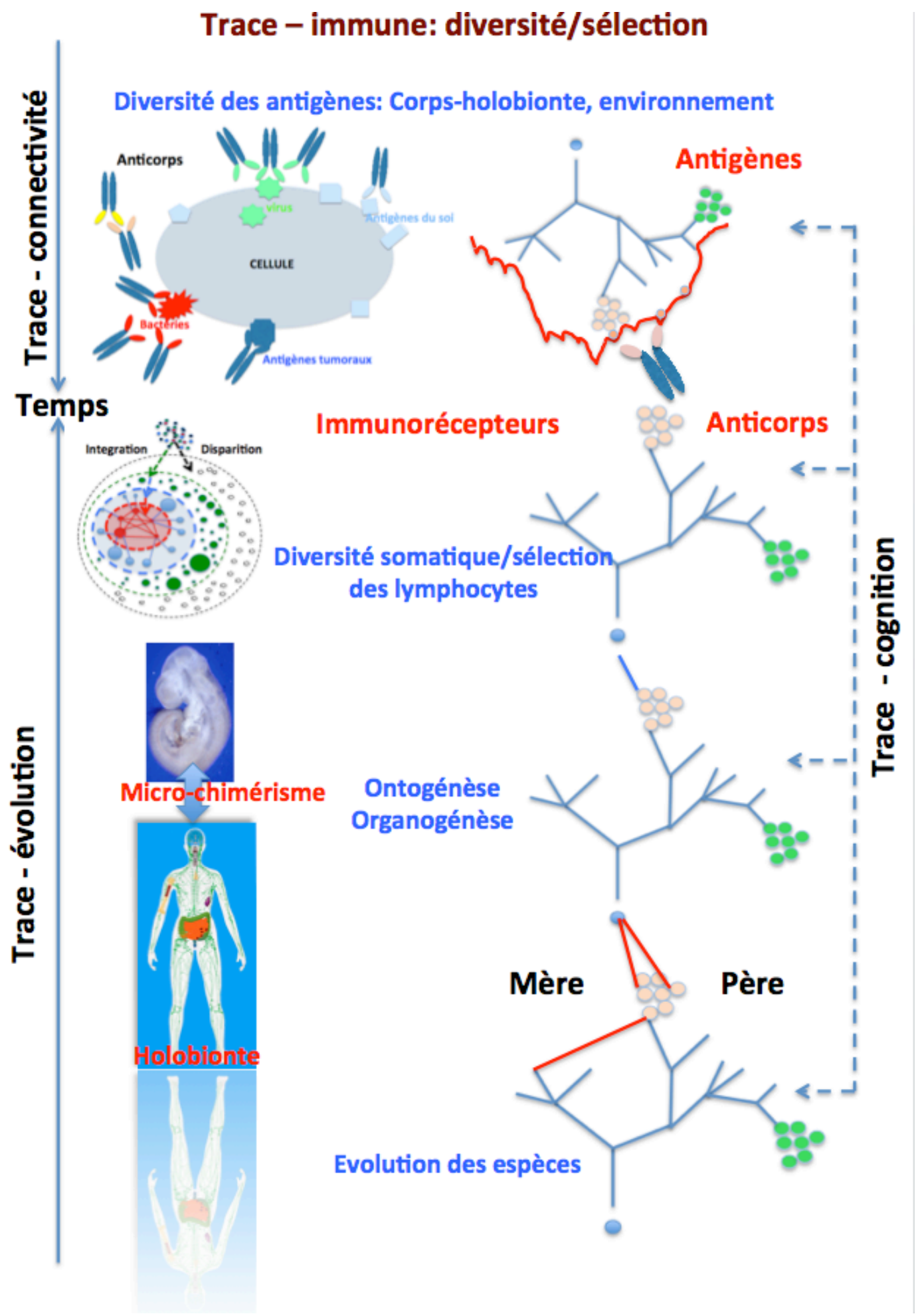

Figure 2.

Au cours de l'évolution des espèces à partir du dernier ancêtre commun (Last Universal Common Ancestor, LUCA), durant des millions d'années, les entités et processus biologiques symbiotiques ont été assemblés, organisés et sélectionnés progressivement pour aboutir à des cellules se reproduisant avec des variations (symbolisées par différentes couleurs). Cette auto-organisation d'entités et de processus est liée à la reproduction, la diversification, les réparations incertaines et mutations au cours des descendances. La croissance des espèces a commencé dans des espaces très restreints, unicellulaires. L'immunité adaptative est apparue dès les bactéries, a évolué et s'est complexifiée par la suite dans les autres espèces. Des symbioses entre espèces (trait 
rouge) ont permis l'apparition de mitochondries dans des cellules eucaryotes (traceur de la transmission maternelle). Puis l'assemblage coordonné dans l'espace et le temps a permis l'organisation d'organismes plus gros, pluri-cellulaires, pluri-génomiques jusqu'aux mammifères. La fécondation sexuée permet la transmission d'informations des deux parents dans les cellules somatiques, mais aussi d'informations uniparentales (l'ADN mitochondrial maternel et chromosome Y d'origine paternelle). L'organisation d'individu pluricellulaire a lieu durant l'embryogenèse : à partir d'une cellule unique le zygote se développera en quelques jours ou mois en un organisme qui différencie des organes et croit progressivement pour assurer toutes les fonctions vitales, dont les fonctions hématopoïétiques et immunes (symbolisées par différentes couleurs). Des échanges bidirectionnels de cellules et d'anticorps ont lieu durant la gestation entre la mère et le foctus, permettant la transmission de l'immunité acquise de la mère. La transmission du microbiote hérité de la mère est également importante et crée une trace non génétique, acquise selon Lamarck. Durant l'ontogénèse, le système immunitaire se différencie chez l'homme en générant une diversité somatique des lymphocytes et de leurs immuno-récepteurs. Le réarrangement somatique de l'ADN du récepteur de chaque lymphocyte permet la constitution de séquences protéiques hypervariables (symbolisées par différentes couleurs), spécifiques à chaque lymphocyte. La dynamique de renouvellement intense et sélection des clones de lymphocytes est assurée durant toute la vie: les lymphocytes sélectionnés subissent de fortes expansions alors que les autres meurent pour 95\% d'entre eux. Le répertoire des lymphocytes est complet car il permet la cognition et le déclenchement de réponses immunes face à des antigènes issus de la diversité des espèces (soi, microbiote, aliments, etc.) mais également face aux produits chimiques. Ainsi les cellules présentent des antigènes variés du soi, tumoraux, viraux, bactériens, chimiques qui sont reconnus par les anticorps et récepteurs des lymphocytes. Les lymphocytes prolifèrent pour réguler le nombre d'antigènes proliférant comme dans le cas des microbes. Le système immunitaire, permettant d'assurer l'identité, l'intégrité, la survie et la reproduction, reprend les processus évolutifs et sélectifs des autres échelles. Le répertoire diversifié des immuno-récepteurs est la "trace" interne du monde antigénique invisible présente dans le corps, créant un système arborescent imbriqué et connecté, de type fractal. Le bord spatio-temporel en déformation entre immuno-récepteurs et celui des antigènes est symbolisé par la ligne rouge discontinue ${ }^{20}$.

Le système immunitaire existe chez tout être vivant, y compris les plantes et les bactéries, bien que des stratégies diverses soient apparues indépendamment durant l'évolution des espèces, et maintenant dans les systèmes immunitaires artificiels construits par les chercheurs (Cooper, 2010). Ainsi, chez les bactéries comme E. Coli, une immunité adaptative et une mémoire des infections sont liées à l'expression de CRISPR (Clustered Regularly Interspaced Short Palindromic Repeats), permettant par interférence ARN d'intercepter le matériel génétique de plasmides et de phages qui infectent la bactérie et de neutraliser une infection ultérieure. Le système immunitaire qui a évolué conjointement aux espèces est devenu chez le mammifère un système dynamique, cognitif, adaptatif et anamnestique qui permet de faire face à des évènements moléculaires et microbiens stochastiques et imprévisibles et d'assurer la survie de l'espèce. Globalement, les réseaux biologiques dépendent d'une organisation complexe mêlant stochasticité et historicité, qui imposent des contraintes au système. Il n'y a pas de déterminisme de la trajectoire des cellules du système immunitaire, des organismes, de la société, ni de l'écosystème.

\section{Le corps holobionte et la transmission de traces}

La trace-transmission, l'historicité liée au vivant, n'est pas que génétique, ni unidirectionnelle vers la descendance, mais associe différents processus mis en place entre les espèces puis dans l'organisme 
holobionte. La transmission d'informations a lieu entre générations, elle est de nature génétique, bi- ou monoparentale, épi-génétique ou acquise par l’expérience. Selon Larmarck :

$$
\text { « } 4 \text { loi: Tout ce qui a été acquis, tracé ou changé dans l'organisation des }
$$

individus pendant le cours de leur vie, est conservé par la génération, et transmis

$$
\text { aux nouveaux individus qui proviennent de ceux qui ont éprouvé ces }
$$

changements ».

Nos organismes humains, comme la plupart des organismes vivants, sont des holobiontes caractérisés par la présence d'un microbiote, indispensable à notre vie (Bordenstein and Theis, 2015). On observe aujourd'hui la trace de symbiose entre entités vivantes de différentes origines dans toute forme de vie (Figure 3). Une mitochondrie interne à la cellule est ainsi issue de la sélection d'une cellule infectée par une bactérie «externe ». L'ADN ou génome mitochondrial représente la trace de cette infection et internalisation il y environ deux milliards d'années, avec une transmission maternelle exclusivement (trace maternelle mitochondriale). La transmission du chromosome $\mathbf{Y}$ est lui paternel. Ces sont des traceurs génomiques utilisés par les chercheurs pour étudier les généalogies et migrations humaines (Underhill et Kivisild, 2007).

\section{Le microbiote}

Notre microbiote (la flore intestinale, muqueuse et cutanée) compose environ la moitié des cellules de l'organisme (soit de l'ordre de $3.8 \times 10^{13}$ bactéries et $3.0 \times 10^{13}$ cellules humaines dont $1.5 \%$ sont des lymphocytes et $90 \%$ sans ADN comme les globules rouges) (Sender, Fuchs et al., 2016) et 90\% de son ADN. Les bactéries commensales sont indispensables à notre vie, notamment pour la digestion, comme en témoigne par exemple la synthèse de la vitamine K. Le microbiote initial est essentiellement transmis au nouveau-né par la mère lors de l'accouchement. Cette transmission (trace-microbiote) est nécessaire au bon développement du microbiote de l'enfant durant les deux premières années, à sa digestion et à l'éducation du système immunitaire. Le microbiote est également spécifique aux espèces et au mode alimentaire (Ley, Lozupone et al., 2008). 


\section{Le corps holobionte Agrégation de molécules et de cellules d'origines polygénomiques}

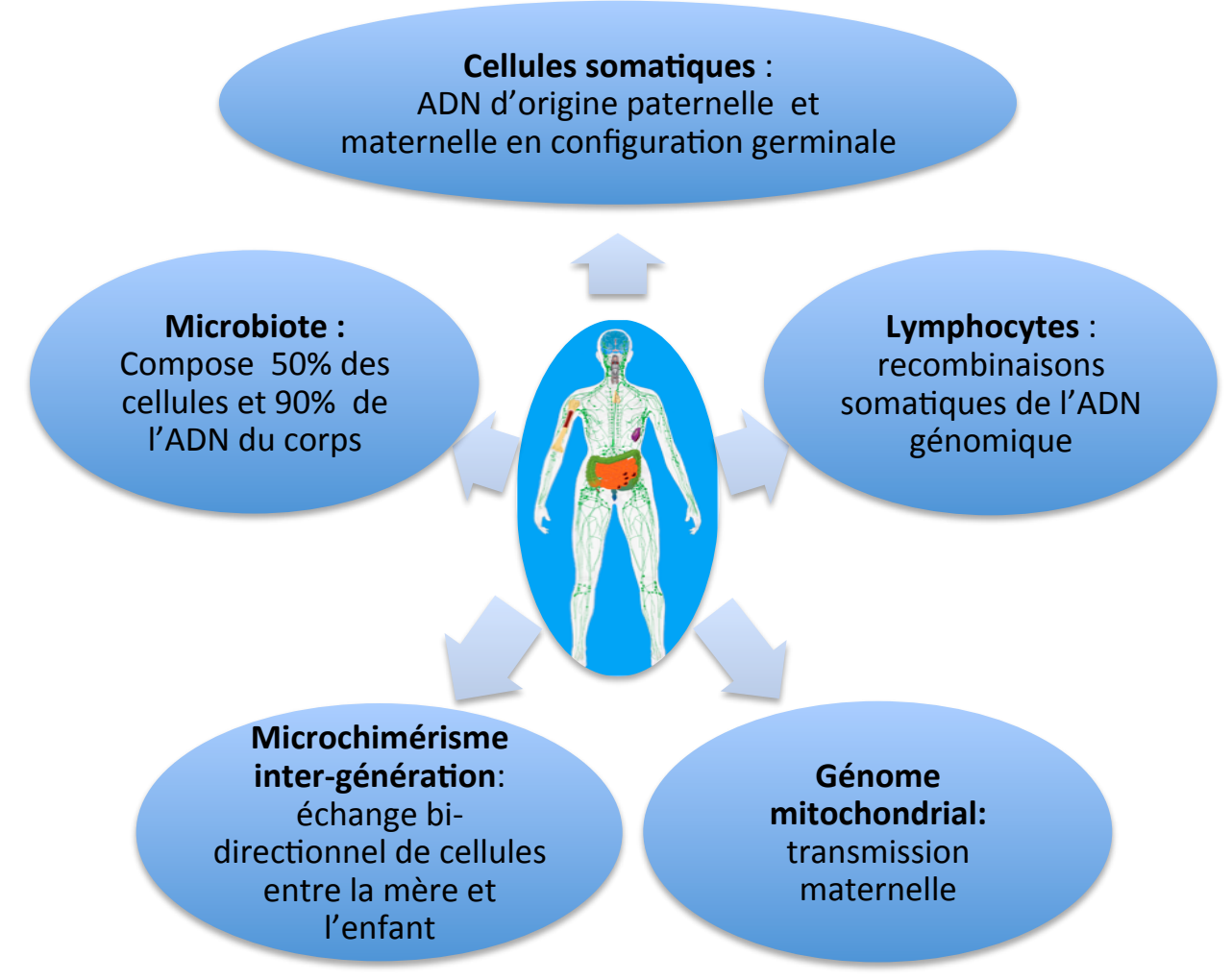

Figure 3.

Illustration du corps holobionte et polygénomique dont les éléments sont des signestraces résultants de processus de la transmission, la trace d'héritages génétiques (cellules somatiques, génome mitochondrial), de diversification somatique du génome dans les lymphocytes, de transmission de caractères acquis tel le microbiote, et de microchimérisme materno-foetal.

La diversité du microbiote sélectionné au cours de l'évolution dans la symbiose hôte-microbes est en équilibre avec le système immunitaire pour garantir un état de bonne santé. En effet, le microbiote oriente les réponses du système immunitaire et la régulation de la tolérance via les lymphocytes T régulateurs (Ivanov et Littman, 2011 ; Kinoshita et Takeda, 2014). Ainsi, le contact précoce des enfants avec certaines bactéries protectrices sélectionnées durant l'évolution permet l'orientation correcte du système immunitaire, et limite le développement d'allergie, d'auto-immunité et de l'obésité. En revanche, l'altération de certaines «traces-microbiote » peut altérer la santé du corps et en conséquence la santé des sociétés humaines. Des «traces processuelles disruptives ou perturbatrices» peuvent donc changer l'orientation du système immunitaire et conduire à des pathologies, ou au contraire à des traitements.

\section{Micro-chimérisme et traces materno-fotales}

Chez l'homme, au cours de l'ontogénie (Figure 2), le fœtus interagit avec sa mère par des transmissions bidirectionnelles, qui participent à la construction du réseau immunitaire. Des échanges de cellules et molécules provoquant un micro-chimérisme cellulaire et moléculaire existent entre la mère et le fœus. 
Selon Lamarck, cette trace inter-générationnelle permet la transmission de caractères acquis. Le répertoire des anticorps maternels est en effet porteur «d'informations» acquises en fonction de l'histoire immunologique de la mère qui peut être transmise au fœtus (Lemke, Coutinho et al., 1975). Cette transmission d'anticorps favorise une meilleure réponse contre des agents infectieux mais peut également participer à une transmission d'auto-immunité. Inversement, certaines cellules fœtales peuvent être transmises et tracées chez la mère (par exemple via la présence de chromosome Y pour les femmes qui ont eu un garçon). Ces cellules d'origine fœtale participent à la réparation de tissus de la mère, mais les lymphocytes peuvent aussi engendrer des réactions d'auto-immunité chez la mère (Nelson, 2012).

\section{Diversité des répertoires des immuno-récepteurs et des antigènes}

La diversité est exemplaire et unique dans le cas de l'organisation du système immunitaire face à l'infinité des antigènes existants : chaque lymphocyte diversifie individuellement au niveau somatique, par des réarrangements de gènes, la séquence de son $\mathrm{ADN}$ génomique codant la région hypervariable de son immuno-récepteur. Chaque lymphocyte présente donc un signe-trace-ADN somatique hypervariable (correspondant à une séquence nucléotidique). Cela permet l'expression d'un immuno-récepteur protéique avec une séquence unique d'acides aminés dans chaque lymphocyte T ou B (Figure 2). Chaque immunorécepteur présente donc une «molécule à région variable» (VRM) (Stewart, 1994), capable par dégénérescence d'interagir avec la multitude de molécules également très variables que sont les antigènes (Figure 2). L'ensemble des lymphocytes diversifiés crée un répertoire somatique, qui représente un signetrace de l'histoire immunologique de l'individu. Le répertoire des lymphocytes diversifiés estimé à environ $10^{10}$ séquences différentes est donc capable de reconnaître le répertoire des antigènes diversifiés estimé à $10^{17}$. Le répertoire des lymphocytes, sorte d'empreinte immunologique des antigènes qui ont interagi avec les lymphocytes, est actuellement étudié afin de caractériser sa variabilité (Six, Mariotti-Ferrandiz et al., 2013 ) et de caractériser les pathologies inflammatoires et auto-immunes ${ }^{14}$.

Le système complexe est fonctionnel au niveau de l'organisme grâce à cette diversification, la connectivité en réseau dynamique, des circuits dégénérés (Edelman et Gally, 2001) permettant à des structures différentes d'arriver à des fonctions différentes ou identiques selon le contexte.

\section{Sélection des lymphocytes et apoptose}

Les lymphocytes subissent une sélection drastique qui dépend des contraintes de l'environnement : il existe une amplification clonale et identique (redondance) de certains lymphocytes qui entre en cognition d'antigènes, alors que plus de $95 \%$ des lymphocytes sont éliminés régulièrement durant la différenciation et sélection thymique pour les lymphocytes $\mathrm{T}$, puis lors de la fin de la réponse immunitaire : une apoptose massive permet de contracter le système pour faire face aux contraintes d'espace dans l'organisme et permettre le renouvellement du système immunitaire et la sélection permanente des lymphocytes (Figure 1). Cette apoptose est aussi une trace-processuelle de re-modelage de divers tissus dans l'organisme durant la morphogénèse (par exemple la séparation des doigts qui peut être incomplète).

L'apoptose est une trace processuelle ordonnée qui commence par la condensation de la chromatine au niveau du noyau de la cellule, la fragmentation de l'ADN, la formation de corps apoptotiques, et finalement la phagocytose par les macrophages, ne créant pas d'inflammation. On peut ainsi trouver la trace d'une à cinq cellules en apoptose à l'intérieur des macrophages qui nettoient le thymus des cellules mortes, du fait d'une importante sélection. La mort cellulaire par nécrose suite à des lésions mécaniques ou chimiques induit quant à elle l'éclatement de la cellule et des processus inflammatoires. On dispose donc de «signetrace» permettant de qualifier et quantifier la mort des cellules et des lymphocytes par apoptose ou nécrose, qui sont des informations importantes permettant d'orienter différemment les réponses du système immunitaire.

\section{Immunoception, cognition et communication}

Le système immunitaire est, dans l'organisme holobionte, à l'interface entre diverses formes vitales hétérogènes et de l'environnement. Le système immunitaire forme ainsi un système sensoriel cognitif, mais différent et complémentaire du système nerveux, avec dix fois plus de cellules (de l'ordre de $10^{12}$ lymphocytes chez l'homme) qui circulent en permanence et sont capables de percevoir des stimuli et informations de l'environnement interne ou externe de l'organisme vivant. Les cellules de l'immunité innée, les plus anciennes dans l'évolution des espèces, sont capables à l'aide de capteurs (Pattern Recognition Receptors), de reconnaître des motifs structurels conservés (signes-trace) et partagés par des 
classes de microorganismes (Pathogen-Associated Molecular Patterns), ou des traces fonctionnelles (traces-processuelles) liées à la destruction de tissus par des pathogènes ou par la réponse du système immunitaire, permettant en retour la réparation ou la réponse immunitaire (Medzhitov, 2013) (Iwasaki et Medzhitov, 2015). Les cellules saines émettent des signes de leur santé et intégrité, ce qui inhibe leur destruction. En revanche, des cellules altérées par une infection, le vieillissement ou une nécrose envoient des signaux d'alarme ce qui permet d'enclencher des processus de réparation, de mort cellulaire programmée par un signal (apoptose), ou la phagocytose des cellules endommagées par les macrophages (Medzhitov et Janeway, 2002). Les cellules de l'immunité adaptative, les lymphocytes apparus plus tard dans l'évolution avec les vertébrés à mâchoire, permettent une perception spécifique, une identification des antigènes. Nous proposons ici de nommer cette perception 1'《immunoception» (Thomas-Vaslin, 2016). Elle se définit comme la perception par les immuno-récepteurs hypervariables, du monde moléculaire invisible qui entoure les lymphocytes, incluant le système immunitaire lui-même. Du fait de cette cognition, le corps contient globalement une représentation interne, un signe-trace particulier, la traceantigène de l'environnement vécu, senti par l'ensemble des lymphocytes en interaction, via les immunorécepteurs spécifiques portés par chaque lymphocytes et les anticorps sériques qui reconnaissent divers «corps» microscopiques. Les lymphocytes et anticorps captent donc et mémorisent qualitativement et quantitativement la trace-processuelle de la cognition des antigènes (trace-antigène) et de leur évolution dans le temps. L'immunoception contribue ainsi à la connaissance moléculaire de notre environnement, par une sorte de langage, de phrases, comme proposé par le prix Nobel N.K. Jerne (Jerne, 1985), lié à l'occurrence des régions hypervariables portées ou secrétées par chaque lymphocyte et la conformation tridimensionnelle des chaînes protéiques les composant. Les immuno-récepteurs diversifiés sont euxmêmes des antigènes, et constituent le réseau idiotypique mis en évidence par Jerne.

La notion de sémiotique en immunologie peut être utile afin de révéler la communication entre cellules avec des signes et codes permettant l'interprétation de signaux dans le système immunitaires (Eco, 1988). La communication inter-cellulaire utilise des combinaisons de récepteurs cellulaires qui réceptionnent des combinaisons de ligands solubles. Ainsi, des combinaisons de cytokines orientent la différentiation et les fonctions effectrices des lymphocytes, et des combinaisons de chimiokines attirent les lymphocytes à distance et orientent leur migration. Les combinaisons qualitatives et quantitatives d'antigènes, de cytokines et de chimiokines présents dans l'environnement des lymphocytes peuvent être des «signes» d'un contexte inflammatoire ou infectieux. Lors de la liaison de ces combinaisons de molécules qui représentent un «lexicon» avec des récepteurs spécifiques à la surface du lymphocyte qui représente un système «d'écoute », elles transmettent un signal. La transduction du signal dans le lymphocyte jusqu'au noyau provoque la synthèse de nouvelles molécules (comme des anticorps par les lymphocytes B, ou d'autres cytokines), qui sont elles-mêmes d'autres signes-traces du processus d'activation du lymphocyte et vont influencer son comportement. Les cellules peuvent ainsi communiquer spécifiquement et connaître l'état de leur environnement. Les synapses immunologiques qui se forment de façon transitoire entre les cellules de l'immunité permettent l'intégration de signaux et la communication par des échanges d'informations. On peut identifier et modéliser ces phénomènes (Souza-e-Silva, Savino et al., 2009 , AbouJaoude, Monteiro et al., 2014).

La représentation de l'environnement interne et externe de l'organisme est liée à la fonction d'endocytose des cellules permettant d'internaliser des molécules ou des microorganismes. Des « cellules présentatrices d'antigènes » telles que des macrophages et des cellules dendritiques phagocytent et présentent les antigènes fragmentés aux lymphocytes T pour les activer. Il existe ainsi des épitopes ${ }^{21}$ publics, communs à un ensemble d'individus, ou privés (Welsh, 2006). L'interaction entre cet environnement miniaturisé, cette présentation de motifs d'antigènes par les cellules présentatrices et les lymphocytes qui entrent en cognition montre qu'il existe une communication interne sophistiquée, bien que le corps n'en ait pas conscience, et dont on peut étudier des signes-traces. Comme souligné par Galinon-Mélénec, «L'hypothèse de l'imbrication entre corps affect, cognition [...] témoigne qu'il existe des relations complexes entre ces composantes et la communication». Ici on montre que les trace-immunes doivent également être considérées dans ces processus de communication du corps par rapport à lui-même et son environnement. D'ailleurs ces traces processuelles créent des patterns spatiaux et temporels, reconnaissables au sein des tissus par des agrégations ordonnées de cellules créant des structures liées à la dynamique d'interaction et de fonctions, qui peuvent être étudiés comme des signes-traces en histologie ${ }^{22}$.

De nombreuses pathologies comme les inflammations, les maladies auto-immunes, les allergies, les infections, les tumeurs, le diabète et le vieillissement présentent des altérations de ces combinaisons ${ }^{23}$ de

\footnotetext{
${ }^{21}$ Les épitopes sont des parties d'antigènes qui ont été coupés et ces motifs (des signes-trace) sont reconnus par les
} immuno-récepteurs.

${ }^{22}$ L'histologie est étymologiquement le discours des tissus.

${ }^{23} \mathrm{Cf}$. Ilangumaran et Ferbeyre (2016). 
signes-traces. Ces molécules de communication et leurs récepteurs sont de potentielles cibles pour des interventions thérapeutiques. Par exemple, de faibles doses d'interleukine-2 permettent dans des cas d'autoimmunité et d'inflammation de réduire l'activation des lymphocytes effecteurs provoquant les pathologies, en activant des lymphocytes régulateurs et donc en rétablissant une balance délicate de communication entre cellules et molécules (Klatzmann et Abbas, 2015).

L'origine et le devenir de notre corps dépendent donc de traces-processuelles dynamiques, évolutives, cognitives, immunes, anamnestiques et émergentes, mêlant différents niveaux spatio-temporels.

L'environnement du corps et son comportement (sommeil, activité physique, stress, etc.) influencent les réponses immunes qui peuvent être délétères sur le système nerveux avec un effet retour sur le comportement. Le stress peut avoir des origines multiples. Il constitue un signe-trace particulier (tracestress). Or le système immunitaire est en communication effective avec le système nerveux (Steinman, 2004) et endocrinien via des neurotransmetteurs, des hormones neuro-endocrines, des cytokines. Un stress peut causer un processus susceptible de provoquer l'effondrement rapide de l'immuno-surveillance, une immunodépression qui favorise les pathologies telles que les infections, le cancer, les métastases (Reiche, Nunes et al., 2004) et le vieillissement (Thomas-Vaslin, Six et al., 2012) qui, dans cette perspective, deviendraient des «signes-traces » résultants : trace-infection, trace-cancer, trace-vieillissement dont on observe des signatures biologiques particulières que ce soit au niveau des populations cellulaires, mais aussi au niveau épigénétique - des altérations des méthylations de l'ADN (Yuan, Jiao et al., 2015), du raccourcissement des télomères peuvent être la trace de stress, d'infections chroniques et de l'âge (Effros, 2011).

$\mathrm{Au}$ contraire, l'activité physique et le sport semblent favoriser l'activité, le métabolisme et le renouvellement dynamique et augmenter la régénération des télomères des populations cellulaires du système immunitaire et leur diversité en diminuant l'inflammation (Simpson, Lowder et al., 2012). L'efficacité du système immunitaire à réagir aux diverses perturbations rencontrées va contribuer au comportement global du corps, définir l'immunocompétence ou non de l'individu, son état de santé, et influencer ses décisions et interactions sociales et culturelles, qui en retour, influence la trace-immune globale. Il s'agit donc d'une récursivité des phénomènes. Comprendre, connaître, préserver et contrôler son système immunitaire et ses réactions par une influence du système nerveux, du rythme circadien (Cermakian, Lange et al., 2013) du sommeil et des activités, des oscillations biologiques (trace-oscillation) mais aussi de l'environnement, de la diversité de la nourriture, du microbiote, des thérapies (vaccinations, anti-inflammatoires, immuno-suppresseurs, antibiothérapie, etc.) peut permettre de mieux vivre son corps et les «traces immunes» qui y sont mémorisées. L'historicité et la mémoire immunologique qui apparaissent au cours du temps biologique, sont particulières aux systèmes vivants pour transmettre la « trace » de processus "sélectionnés" au cours du temps qui s'appuie sur le système immunitaire de chaque individu. Certains processus dynamiques anamnestiques permettent la transmission d'informations, via le système immunitaire, en dehors de l'héritage génétique.

\section{Connectivité idiotypique des immuno-récepteurs}

Chez le fœtus, la connectivité des immuno-récepteurs procède progressivement dans l'espace et le temps (Andrade, Huetz et al,. 1991, Thomas-Vaslin, Andrade et al., 1991) (Figure 4). Les premiers lymphocytes (en rouge) génèrent une classe d'immuno-récepteurs, appelées les immunoglobulines naturelles IgM, qui sont multi-réactives et auto-réactives (Freitas, Pereira et al., 1989). Ces immunoglobulines IgM ont des interactions mutuelles avec d'autres anticorps ce qui provoque une cognition idiotypes/anti-idiotypes (les anticorps sont eux-mêmes des antigènes) qui forme le réseau idiotypique proposé par Jerne (Jerne, 1984). Ces immuno-récepteurs très connectés forment un «noyau dynamique» central interactif au sein du réseau moléculaire (central immune system - CIS) (Thomas-Vaslin, 2014). Ici participent aussi les immunoglobulines transmises par la mère (son propre réseau idiotypique déjà sélectionné qui a une mémoire). L'ensemble contribue à l'intégration connective des nouveaux lymphocytes produits durant toute la vie (Peripheral Immune System - PIS) qui vont survivre ou non selon la séquence protéique hypervariable de leur immuno-récepteur. Les lymphocytes générés après la naissance n'ont pas autant de connectivité. Ils sont plus dépendants de la présence de l'antigène spécifique pour leur survie (en vert), et les clones non stimulés disparaissent (en gris) (Figure 4). 


\section{Intégration des lymphocytes dans le réseau immunitaire}

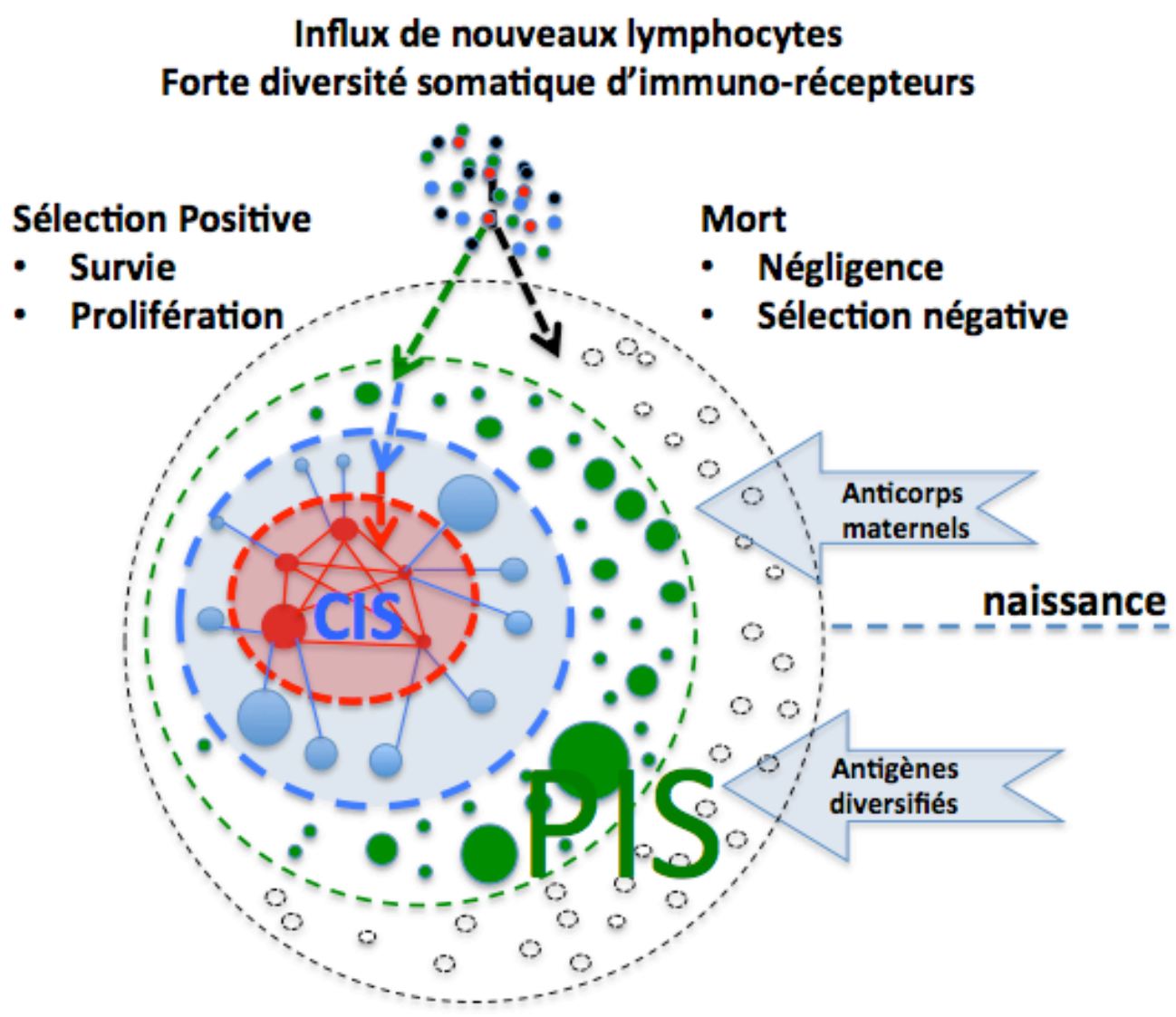

Figure 4.

Schématisation de l'organisation du réseau d'immuno-récepteurs et de leur connectivité (réseau idiotypique) : chaque nouveau lymphocyte produit (symbolisé par des clones de différentes couleurs et tailles) est sélectionné. Les lymphocytes du système immunitaire central (CIS) sont très connectés, auto-réactifs et multi-réactifs (clones du noyau central en rouge) et fortement présents avant la naissance, reconnaissant les antigènes $d u$ "soi » et les anticorps maternels transmis. Ils sont donc activés et prolifèrent. L'intégration dans le système immunitaire périphérique (PIS) (clones en vert) de nouveaux lymphocytes diversifiés (microbiote, infections...) dépend de la capacité des cellules à s'intégrer dans le réseau, à réagir à des antigènes plutôt " externes » au corps, sinon ils meurent par apoptose (clones en pointillés) (Thomas-Vaslin, 2014a).

Environ $15 \%$ des lymphocytes sont naturellement auto-réactifs chez l'adulte et sont régulés par des lymphocytes régulateurs (boucle de contrôle négative) pour maintenir la tolérance et éviter des pathologies auto-immunes ou inflammatoires. La durée de vie et la prolifération des lymphocytes n'est pas déterminée mais dépend de leur sélection et interaction continuelle avec l'environnement via leur immuno-récepteur spécifique (Thomas-Vaslin, 1990).

\section{Cognition moléculaire des antigènes par les anticorps et}

\section{comportement du corps}

Comme nous l'avons vu, la trace-processuelle se définit comme le processus d'une émergence dynamique qui percole d'autres processus depuis d'autres niveaux d'échelle laissant des signe-trace. On peut alors concevoir l'existence de signe-trace-émergents et de certaines régularités. Ainsi au niveau individuel et 
social, le résultat de perturbations immunitaires est visible par des occurrences de certaines pathologies en augmentation sensible, et en retour des modifications du comportement social et culturel influencent le comportement du système immunitaire et l'occurrence de pathologies. On peut aussi parler de «trace récursive » dans le temps (entre générations) mais aussi dans l'espace du corps entre les cellules du «soi » et du microbiote (la consistance interne) et dans l'espace de l'environnement puisque selon Nicolas Bouleau, tout organisme agit sur l'environnement (consistance externe) ${ }^{24}$.

La dynamique des processus permet l'émergence de la régularité des mesures observées et des corrélations fonctionnelles, temporelles et spatiales entre des paramètres dans un réseau multidimensionnel et dynamique, si les contraintes et sélections sont semblables. Cette organisation crée de l'anti-entropie (Bailly et Longo, 2009), de la complexité dynamique qui est une sorte d'organisation du désordre pour créer de l'ordre au cours du temps et limite certains choix mais maintient aussi l'ordre produit au cours du temps. On observe ainsi des signatures (ou «patterns») par la qualification et quantification de biomarqueurs, soit dans des mesures synchroniques ou diachroniques. Ces signatures constituent des sortes d'invariants qui sont l'empreinte, la trace de l'historicité de l'espèce à travers des stabilités macroscopiques (structure et organisation générale, anatomie) ou microscopiques (séquence du génome). Pourtant, il existe une grande variabilité fonctionnelle, même au sein du même organisme au cours du temps. Le regroupement statistique des observables selon leur distance permet d'observer des arborescences, des clusters dans ces signatures, de mesurer leur variabilité et de retracer leur évolution à travers ces variations. Les invariants en biologie et en immunologie ne sont pas des entités et des structures (comme décrit en physique), mais des processus et des fonctions de métabolisme, reproduction, réparation.

L'identité immunologique est variable : le répertoire des lymphocytes, c'est-à-dire l'ensemble des régions hypervariables générées au niveau somatique, varie au cours du temps. Le répertoire des antigènes dits « du soi » varie selon les tissus (Thomas-Vaslin, Salaun et al., 1995) et selon des modifications épi-génétiques. Des dé-méthylations locales de l'ADN permettent une expression ectopique de gènes dans certaines cellules (Derbinski, Schulte et al., 2016). La trace ou l'empreinte de chaque tissu est donc spécifique. Ainsi dans un système de greffe de tissus provenant du même donneur, alors que des greffes de peau ou de cœur peuvent être tolérées, la greffe de lymphocyte B est rejetée. En effet, les idiotopes (partie hypervariables des lymphocytes) sont la trace spécifique de chaque individu et de son histoire, de son vécu, et sont « incompatibles» avec un autre individu pourtant génétiquement identique. À partir d'un répertoire de lymphocytes au départ aléatoire (désordre), on observe ainsi en laboratoire des répertoires de lymphocytes similaires dans la rate des souris jeunes de même fond génétique, élevées dans le même environnement (ordre). Pourtant, on peut noter qu'avec le temps le système immunitaire de jumeaux homozygotes diffère de $80 \%$, montrant que chaque individu, chaque corps construit sa propre "trace immune» indépendamment de l'héritage génétique (Brodin, Jojic et al., 2015) au cours de sa vie. La variabilité des répertoires est plus importante chez les individus plus âgés où du désordre apparaît avec la trace processuelle du temps biologique (signe-trace vieillissement), selon le vécu du corps, face aux divers antigènes rencontrés et des désordres multi-échelles qui apparaissent (Thomas-Vaslin, Six et al., 2012). En particulier, des populations de lymphocytes CD8 peuvent subir des expansions par sénescence cellulaire ou disparitions de clones (Thomas-Vaslin, Six et al., 2012). La trace de l'accumulation de ces clones CD8 sénescents, observés dans différentes espèces de mammifères, peut être liée par exemple à des stimulations chroniques, comme l'infection chronique par des virus. La réponse immune contre le cytomégalovirus peut être un élément pronostique de l'augmentation de l'incidence de mortalité chez l'homme âgé (Hadrup, Strindhall et al., 2006). De même les signatures liées aux bio-marqueurs observées dans un état de "bonne santé" sont altérées en cas de vieillissement ou de pathologie. On peut alors questionner la perte d'efficacité du métabolisme et des processus de réparation sous-jacents dans les cellules mais aussi les altérations de prolifération ou mort cellulaire, qui provoquent l'altération des traces émergentes et processuelles révélées au niveau des populations cellulaires des tissus et finalement de l'organisme, et marque le corps de l'accumulation de perturbations liées au temps (Tableau 1).

\section{Mémoire, trace-mnésique}

La trajectoire des lymphocytes, leur dynamique et sélection n'est pas déterminée mais se construit, influencée par environnement (Thomas-Vaslin, 1990). Grâce à son évolution permanente, la trace-immune garde une mémoire immunologique, la trace-mnésique naturelle ou provoquée, post-vaccinale. Cette mémoire confère au système la capacité de répondre plus efficacement, plus vite aux antigènes que lors de la première rencontre et avec une plus grande amplitude après une primo-infection ou une vaccination.

\footnotetext{
${ }^{24}$ Bouleau N., L'environnement providence, 2015, https://halshs.archives-ouvertes.fr/halshs-00822986.
} 
Cette capacité anamnestique immunologique adaptative est le produit de la sélection des espèces, qui apparaît déjà chez les bactéries comme E. Coli dans les «clustered regularly interspaced palindromic repeat », utilisé maintenant en biotechnologies pour l'ingénierie du génome ${ }^{25}$ (Doudna et Charpentier, 2014). À partir des vertébrés à mâchoire, un système immunitaire adaptatif différent émerge permettant la diversification somatique des immuno-récepteurs des lymphocytes donc une possible sélection parmi une diversité de lymphocytes (Flajnik et Kasahara, 2010). Les animaux «immunisés » sont donc sélectionnés car ils ont résisté, sont plus robustes et conservent la mémoire, la trace de l'expérience antérieure acquise et survivent mieux à une infection similaire subséquente. Il ne s'agit pas de système anticipatif (comme proposé par Rosen) mais de sélection et d'activation parmi une diversité de possibles, qui ont déjà été restreints par des expériences passées, conférant une historicité aux systèmes vivants. Chez les mammifères, cette mémoire dynamique est représentée par le réseau des immuno-récepteurs (les récepteurs aux antigènes) et des lymphocytes sélectionnés au cours du temps. Les caractéristiques du répertoire sont le signe-trace des rencontres temporelles avec les antigènes, des signaux intracellulaires transmis au noyau cellulaire et des régulations positives et négatives qui ont eu lieu. Ainsi la mémoire immunologique, transmise passivement au fœtus via les immunoglobulines de la mère ou acquise durant l'enfance (infections, vaccinations), est la trace de rencontres antigéniques qui augmentent la réactivité et efficacité du système (Tableau 1).

Le maintien de la mémoire immunologique est plutôt lié à la division des lymphocytes $\mathrm{T}$ (donc une trace dynamique interactive) qu'à leur survie en état de quiescence (Bellier, Thomas-Vaslin et al., 2003). Cette efficacité est liée à la réactivation et la prolifération rapide et compétitive de clones de lymphocytes spécifiques des antigènes, préalablement sélectionnés, activés et qui en gardent la trace. Cette mémoire individuelle à chaque lymphocyte et collective pour l'ensemble des lymphocytes contribue à l'échelle supérieure à l'augmentation de la viabilité des individus par rapport à des individus qui n'auraient pas été immunisés ou vaccinés. La mémoire immunologique permet non seulement de réagir plus vite à des pathogènes mais également de maintenir un état d'activation de cellules régulatrices qui maintiennent un état de tolérance afin de contrôler les lymphocytes auto-réactifs. L'activation précoce de lymphocytes régulateurs auto-réactifs au cours des réponses immunitaires et la mémoire de cette activation permettent d'établir la tolérance au sein du fœtus chez le mammifère, et la tolérance du système immunitaire de la mère face au fœtus, donc d'aboutir à la reproduction de l'espèce. En revanche, cette tolérance (et la mémoire associée) permet aussi la tolérance au développement de tumeurs par une activation rapide des lymphocytes régulateurs, au sein de la tumeur, qui bloquent l'activation des lymphocytes anti-tumoraux (Nehar-Belaid, Courau et al., 2016).

Les mutations des cellules permettant les apparitions de nouvelles espèces, de sous-espèces, de variants, ainsi que la diversité des immuno-récepteurs ne sont pas des anticipations finalisées pour permettre aux générations futures de s'adapter à l'environnement, mais une sélection parmi un choix de possibles, permettant à l'organisme de vivre et se reproduire s'il a la capacité de répondre aux contraintes du système (qui elles-mêmes évoluent), ou à disparaitre dans le cas contraire.

\section{Viabilité multi-échelle}

Il existe une adaptation de chaque nœud de réseau (molécule, cellule, organisme) qui répond aux contraintes et perturbations et qui change de connexion pour tenter de maintenir la cohésion du système dans une zone de viabilité : c'est un espace de phase multidimensionnel où les paramètres biologiques sont possibles, sinon le système est instable, n'est plus viable et disparaît (Aubin, Bayen et al., 2011). L'adaptabilité est une flexibilité qui contribue à la viabilité et la résilience pour maintenir la fonctionnalité du réseau. Les réseaux biologiques et écologiques sont résilients du fait de la diversité des composants (des nœuds), liée à la variation à chaque reproduction (de molécules de cellules ou d'organisme), et de leur sélection (les systèmes non résilients, non adaptés disparaissent). La zone de viabilité des systèmes est donc cet espace de paramètre complexe qui est une trace de la sélection qui a eu lieu. Les traces processuelles, de communication, de réparation, d'adaptation aux contraintes environnementales mettent un certain temps à s'effectuer, à percoler dans un écosystème. Aux niveaux moléculaires et cellulaires, la présence d'antigènes constitue des signaux d'alarme pour activer les cellules de l'immunité innée et adaptative à la pathologie. Des signes d'alarme avant-coureur de bifurcation et de l'effondrement de systèmes peuvent être observés

\footnotetext{
${ }^{25}$ On peut noter ici que cette technologie change l'historité des systèmes et la transmission des traces dans le vivant.
} 
V.Thomas-Vaslin dans «Des Traces du corps au corps-trace», Série L'Homme-trace, tome IV version du 6/4/2017

par des changements d'interaction des réseaux dynamiques de bio-marqueurs dans différentes pathologies, permettant d'avoir ainsi une éventuelle prédiction de l'évolution de pathologies (Chen, Liu et al., 2012) ${ }^{26}$.

\footnotetext{
${ }^{26} \mathrm{La}$ résilience, diversité et viabilité des systèmes complexes vivants multi-échelles, a fait récemment l'objet de questionnement (Thomas-Vaslin, 2014b) et d'un colloque ${ }^{26}$ à l'Institut des Systèmes Complexes de Paris.
} 


\section{CONCLUSION}

\section{Philosophie des traces-immunes et du corps}

Après avoir examiné comment les techniques d'observation portant sur l'organisation multi-échelle des systèmes, de la molécule à l'organisme, permettaient de détecter chez l'Homme des combinaisons de biomarqueurs, «signes-traces du corps », conséquences observables de traces processuelles, éventuellement associées à telle pathologie, nous aboutissons à une mise en perspective de ce chapitre en proposant l'ouverture à la rencontre interdisciplinaire. Cette conclusion nous permettra de repenser le système immunitaire et son rôle dans la résilience des systèmes vivants multi-échelles et organisés dans le corps de chaque individu, mais aussi au niveau de la société.

\section{Repenser les frontières du corps}

Dans la vie de tous les jours et pour la mathématisation et la modélisation des systèmes, il est pratique et courant dans un mode réductionniste d'utiliser des systèmes isolés, comme une boîte noire avec des flux entrant et sortant, interne et externe à une cellule, à un organe (Figure 1) ou un organisme par exemple. Il est ainsi courant de qualifier le système immunitaire de système de « défense » et de discrimination du soi et non-soi, selon une métaphore de discrimination dualiste et guerrière qui perdure encore (Cohn, 2015). Une infection bactérienne dans un organisme ou une infection virale d'une cellule peut être considérée comme externe à l'organisme ou à la cellule (non-soi). Une perturbation telle qu'une mutation dans une cellule, ou la croissance d'une tumeur peut être considérée comme interne (soi) - pour un observateur externe, un patient ou un médecin. Nous sommes des organismes (selon notre perception externe de "soi" et de l'autre) liés à la différenciation de cellules somatiques, issues du zygote après la fécondation, mais surtout un écosystème, un holobionte multi-génomique, symbiotique en équilibre dynamique, face aux perturbations constantes et aux contraintes de l'environnement. Le concept d'holobionte, comme nous l'avons décrit dans son évolution (Figure 2) et ses traces (Figure 3), est caractérisé par une sorte de porosité dans le temps et l'espace de l'individu et de son environnement. Il paraît donc nécessaire de considérer la symbiose, et non la discrimination soi/non-soi ou danger/non-danger, pour définir un organisme qui en réalité n'existe pas en dehors de cette symbiose (Gilbert, Sapp et al., 2012). La dichotomie sujet/objet, soi/non-soi n'existe pas (Tauber, 2008) et devrait être repensée. Il n'existe donc pas formellement d' « individu » ni de «soi ».

Nous venons de voir que le concept de trace est vaste et que les traces sont entremêlées, récursives, et percolent entre les échelles microscopiques et macroscopiques, créant des traces émergentes et immergentes. Ceci justifie le fait qu'il n'y pas d'interne et d'externe au corps, de soi et non-soi et donc de frontière. Notre corps peut être l'objet de perturbations qui ne peuvent être qualifiées d' «internes » ou « externes », mais plutôt d'altération de processus d'interactions globales révélant le déséquilibre de notre écosystème. Ces questions ont fait l'objet d'étude et de débats ${ }^{27}$ en particulier pour le système immunitaire dans (Thomas-Vaslin, 2015a ; Thomas-Vaslin, 2015b).

Avoir une vision holistique et complexe est primordial et nécessite de proposer une organisation biologique dynamique, évolutive, emboîtée et connectée entre de multiples échelles, avec des arborescences temporospatiales anatomiques et fonctionnelles, dans laquelle nos cellules, notre système immunitaire et notre corps évoluent au sein de l'écosystème terrestre. Cette approche complexe multi-échelle fait l'objet de recherches et de modélisations en s'appuyant sur notre compréhension du système immunitaire (Thomas-Vaslin, 2014a ; Thomas-Vaslin, 2015a ; Thomas-Vaslin, 2015b). Une perspective écologique du système immunitaire permet de ne pas isoler le système de son environnement et de proposer une conception holistique plutôt que méréologique (Tauber, 2008).

\section{Regard sur l'émersiologie}

Selon Bernard Andrieu, l'émersiologie représente une écologie corporelle :

\footnotetext{
${ }^{27}$ http://iridia.ulb.ac.be/bersini/Self-NonSelf/index.html http://thomaspradeu.com/research/conferences/conference-redefining-the-self.
} 


\title{
"[...] la langue du corps vivant dans l'éveil de nouvelles sensations jusqu'à la conscience sans que celle-ci l'ait désiré volontairement ni recherché délibérément dans une performance » (Andrieu, 2016).
}

\begin{abstract}
Aussi l'émersiologie considère le sentant de notre corps sensible qui n'est pas le senti. L'émersiologie devrait donc prendre en considération l'interaction du corps, le «sentant » via le système immunitaire.

"Éveiller son corps vivant par des techniques du corps suppose de pouvoir

l'activer en le plaçant dans des situations vivifiantes qui déconstruisent le schéma corporel habituel ».
\end{abstract}

C'est ainsi que l'interaction du système immunitaire et son activation avec notre microbiote, la nourriture, les substances chimiques, les médicaments, la pollution, les vaccins provoquent des traces-immunes qui agissent sur la fonctionnalité immunologique et les réponses immunes conduisent soit au maintien d'un organisme sain, soit à des atteintes pathologiques. Ces réponses immunes sont la trace des processus et des performances immunitaires équilibrées permettant de contenir des agents infectieux, ou de réponses déséquilibrées, conduisant à des pathologies inflammatoires, auto-immunes, le cancer, des infections.

On peut également aller au-delà des propositions initiales de Merleau-Ponty sur la perception du corps et du mental, du visible et de l'invisible, "un système de systèmes voué à l'inspection d'un monde » (Merleau-Ponty, 1969) en incluant le système immunitaire dans cette inspection du monde, comme une «conscience» perceptive de l'invisible, par la sélection de molécules et de lymphocytes capables de percevoir leur environnement et de réagir en conséquence. L'unité du corps et le schéma corporel proposé par Merleau-Ponty peuvent être étendus à l'holobionte, sa diversité et les processus sélectifs qui permettent de maintenir la viabilité du corps, par le métabolisme, des processus de réparation mais aussi les réponses immunes indispensables à notre survie.

\section{Traces-immunes et vieillissement, mode de vie et société}

Les altérations moléculaires intracellulaires liées à la sénescence cellulaire (raccourcissement des télomères, altérations des membranes cellulaires et de la transduction des signaux, mutations, etc.) altèrent les interactions des populations cellulaires et la fonctionnalité des tissus et organes pour finir par émerger par une augmentation de pathologies immunes typiquement liées au vieillissement (inflammation, immunodépression) (Thomas-Vaslin, Six et al., 2012). Inversement la trace du stress et de perturbations ressentis par le corps (antigènes, médicaments, immunosuppresseurs produits chimiques...) influence les niveaux inférieurs (par exemple par l'atrophie rapide des organes lymphoïdes, l'inhibition de la division cellulaire, etc.) et constitue une trace immergente.

Au cours du vieillissement des différents systèmes, de notre corps, de notre société humaine et de notre écosystème, on peut observer aux différentes échelles, des ralentissements du métabolisme, l'accumulation de déchets, des encombrements, des manques de réparations, une baisse de biodiversité. Il se produit alors des pathologies immunes liées à des déséquilibres : pathologies inflammatoires, auto-immunité, augmentation de tumeurs, allergies, infections qui sont des pathologies du système immunitaire. En effet, les changements sociétaux rapides, en quelques décennies, dans nos modes de vie, dans notre alimentation aseptisée, mais dans un environnement envahi de nouveaux produits chimiques organiques ou inorganiques, perturbent le système immunitaire sélectionné lentement au cours de l'évolution. Aujourd'hui plus de 126 millions de produits sont répertoriés et 10000 ajoutés par jour sur le site de l'American Chemistry Society ${ }^{28}$. De nombreux produits chimiques, polluants, allergènes, dioxines, perturbateurs endocriniens, métaux lourds, etc., perturbent le système immunitaire suscitant des allergies, des inflammations, des immunodépressions et des tumeurs (Inadera, 2006).

Selon l'hypothèse de l'hygiène, une hygiène excessive et les antibiotiques chez l'enfant induisent des déséquilibres de la flore intestinale conduisant à des désordres immunitaires et métaboliques à long terme (Ivanov et Littman, 2011 ; Cox, Yamanishi et al., 2014 ; Vatanen, Kostic et al., 2016). Récemment, l'impact de la flore intestinale et de la perte de sa diversité chez des patients, a révélé que ces déséquilibres

\footnotetext{
${ }^{28}$ https://www.cas.org/
} 
sont corrélés à de nombreuses pathologies. L'obésité, le diabète, l'autisme, les allergies semblent être l'émergence récente de perturbations immunes liées au mode de vie moderne, la sédentarité des personnes et l'industrialisation. Les accouchements par césarienne perturbent la flore microbienne sélectionnée au cours de l'évolution pour vivre en symbiose avec les animaux et donc les hommes (Dominguez-Bello, De Jesus-Laboy et al., 2016). L'accouchement par césarienne rompt en effet la transmission de la flore vaginale à l'enfant ce qui peut entrainer des altérations du microbiote et des pathologies immunes et métaboliques au long terme. Cette «trace-microbiote » peut être artificiellement rétablie en transmettant de la flore vaginale aux enfants nés par césarienne (Dominguez-Bello, De Jesus-Laboy et al., 2016).

On peut noter que les techno-sciences capables de modifier rapidement notre environnement et par exemple le génome d'organismes vivants (quelle que soit leur échelle) altèrent la trace historique, processus de l'évolution des êtres vivants et même nient cette historicité. Cela questionne la stabilité de l'organisation de notre écosystème et sa viabilité face à ces perturbations brutales. Récemment la possibilité de faire par ingénierie génétique des modifications géniques dans l'ADN, non seulement des cellules somatiques mais aussi embryonnaires et germinales, qui affecte le patrimoine génétique des cellules reproductives, pose des questions éthiques pour des applications cliniques (Baltimore, Berg et al., 2015). Au-delà de modifications géniques chez l'homme, les organismes génétiquement modifiés participent au changement de traces non seulement dans l'évolution des espèces et leur compétition au sein de l'écosystème, mais aussi modifient la «trace-antigènes » et la réactivité du système immunitaire (Jacquemart, Thomas-Vaslin et al., 2016; Thomas-Vaslin, 2016). Cela pose des questions sur la place des sciences de la complexitée ${ }^{29}$, la résilience et imprédictibilité aux perturbations des systèmes complexes vivants, organisés et interactifs et l'évaluation globale de ces systèmes, pour une éthique, décision et action s'appuyant sur une pensée complexe acceptant l'incertitude ${ }^{30}$.

En effet, ces déséquilibres rapides risquent de sortir le système de sa zone de viabilité et le rapproche de la zone de la frontière de la destruction, de la bifurcation vers un état irréversible. En effet, l'énergie à déployer pour le système pour revenir vers son état de «viabilité » serait trop grande ou nécessitera la combinaison de trop de paramètres qui auront dévié vers des zones inconnues. L'homme invente et innove alors pour contrecarrer ces dysfonctionnements, mais avec une vélocité telle et sur tant de paramètres nouveaux à la fois, que l'adaptation, le rééquilibrage dynamique des fonctions n'ont plus le temps de se réaliser.

En conclusion, après avoir défini quelques exemples de traces générées par le système immunitaire au sein de l'évolution des espèces et de chaque organisme ou utilisées par les chercheurs, on peut proposer de repenser quelques concepts précédemment discutés dans le contexte du corps et de sa perception et de l'effet de la société sur le corps, le système immunitaire et les conséquences pathologiques.

\title{
Références bibliographiques
}

\author{
Abou-Jaoude W., Monteiro P.T., Naldi A., Grandclaudon M., Soumelis V., \\ Chaouiya C. ET ThIEFFry D., «Model checking to assess T-helper cell plasticity», Front
} Bioeng Biotechnol, 2, 2014, p. 86.

\author{
Andrade L., Huetz F., Poncet P., Thomas-Vaslin V., Goodhardt M. et Coutinho \\ A., «Biased VH gene expression in murine CD5 B cells results from age-dependent \\ cellular selection», Eur J Immunol, 21 (9), 1991, p. 2017-2023.
}

\footnotetext{
${ }^{29}$ Thomas-Vaslin, V. Questionnements et connaissance de la complexité. In Editions Matériologiques Paris, Qu'est ce que la science? 2017.

${ }^{30} \mathrm{https}: / /$ www.reseau-canope.fr/congres-mondial-pour-la-pensee-complexe/contributions.html - bandeauPtf. Thomas-Vaslin V., et Jacquemart F. , Approche de la résilience et perturbations des systèmes complexes par une évaluation globale. Congrès mondial pour la pensée complexe: Les défis d'un monde globalisé, 2016.
} 
ANDRIEU, B., Emersiologie. Sentir son corps vivant, Paris, Vrin, 2016.

Aubin J.P., BAYen A. ET SAInt-Pierre P., Viability theory new directions, Berlin, Heidelberg, Springer, 2011.

Bailly F., Longo G., «Biological Organization and Anti-Entropy», J. Biological Systems, $\mathrm{n}^{\circ} 17$ (1), 2009, p. 63-96.

Baltimore D., Berg P., Botchan M., Carroll D., Charo R.A., Church G., Corn J.E., Daley G.Q., Doudna J.A., Fenner M., Greely H.T., JineK M., Martin G.S., Penhoet E., Puck J., Sternberg S.H., Weissman J.S. et Yamamoto K.R., «Biotechnology. A prudent path forward for genomic engineering and germline gene modification», Science, 348 (6230), 2015, p. 36-38.

Bellier B., Thomas-Vaslin V., SARON M.F. et Klatzmann D., «Turning immunological memory into amnesia by depletion of dividing T cells», Proc Natl Acad Sci U.S.A, 100 (25), 2005, p. 15017-15022.

Bersini H., Klatzmann D., SiX A. et Thomas-Vaslin V., «State-transition diagrams for biologists», PLoS One, 7 (7), 2012, e41165.

BordensteIn S.R., THEIS K.R., «Host Biology in Light of the Microbiome: Ten Principles of Holobionts and Hologenomes», PLoS Biol, 13 (8), 2015, e1002226.

Brodin P., Jojic V., Gao T., Bhattacharya S., Angel C.J., Furman D., Shen-Orr S., Dekker C.L., Swan G.E., Butte A.J., Maecker H.T., et Davis M.M, «Variation in the human immune system is largely driven by non-heritable influences», Cell, 160 (1-2), 2015, p. 37-47.

Cermakian N., Lange T., Golombek D., Sarkar D., Nakao A., Shibata S. et MAzzoccoli G., "Crosstalk between the circadian clock circuitry and the immune system", Chronobiol Int, 30 (7), 2013, p. 870-888.

Chen L., LiU R., LiU Z. P., Li M., Aihara K., «Detecting early-warning signals for sudden deterioration of complex diseases by dynamical network biomarkers. » Sci Rep 2: 342, 2012.

CoHN M.. «Analysis of Paris meeting redefining the "Self" of the immune system» Immunol Res, 2015. 
COOPER E. L.. «Evolution of immune systems from self/not self to danger to artificial immune systems (AIS) », Phys Life Rev 7(1), 2010, p. 55-78.

Cox L. M., Yamanishi S., Sohn J., Alekseyenko A. V., Leung J. M., Cho I., Kim S. G., Li H., Gao Z., Mahana D., Zarate Rodriguez J. G., Rogers A. B., Robine N., Loke P. et Blaser M. J., «Altering the intestinal microbiota during a critical developmental window has lasting metabolic consequences» Cell 158(4): 705-721, 2014.

DARWIn, C., On the Origin of Species by Means of Natural Selection, or the Preservation of Favoured Races in the Struggle for Life, Londres, John Murray, 1859.

Derbinski J., Schulte A., Kyewski B. et Klein L,. «Pillars Article: Promiscuous Gene Expression in Medullary Thymic Epithelial Cells Mirrors the Peripheral Self. Nat. Immunol. 2001. 2: 1032-1039. », J Immunol, vol.196(7), 2016, p. 2915-2922.

Derian N., Bellier B., Pham H. P., Tsitoura E., Kazazi D., Huret C., Mavromara P., Klatzmann D. et Six A. «Early Transcriptome Signatures from Immunized Mouse Dendritic Cells Predict Late Vaccine-Induced T-Cell Responses », PLoS Comput Biol, 12(3): e1004801, 2016.

Dominguez-Bello M. G., De Jesus-Laboy K. M., Shen N., Cox L. M., Amir A., Gonzalez A., Bokulich N. A., Song S. J., Hoashi M., Rivera-Vinas J. I., Mendez K., Knight R. et Clemente J. C., «Partial restoration of the microbiota of cesarean-born infants via vaginal microbial transfer. », Nat Med, 2016.

Doudna J. A.,Charpentier E., «Genome editing. The new frontier of genome engineering with CRISPR-Cas9 », Science, 346(6213): 1258096, 2014.

Eco U., «On Semiotics and Immunology. The Semiotics of Cellular Communication», dans Sercarz E. E., Celada F., Mitchison N. A. et Tada T. (dir.), The Immune System. Berlin, Heidelberg, Springer Berlin Heidelberg, 1988, p. 3-15.

Edelman G. M., Gally J. A., «Degeneracy and complexity in biological systems », dans Proceedings of the National Academy of Sciences, U S A 98(24): 13763-13768, 2001.

EFFros R. B., «Telomere/telomerase dynamics within the human immune system: effect of chronic infection and stress », Exp Gerontol 46(2-3): 135-140, 2011.

FLAJNIK M. F., KASAHARA M., «Origin and evolution of the adaptive immune system: genetic events and selective pressures » Nat Rev Genet, 11(1), 2010, p. 47-59. 
Freitas A., Pereira P., Huetz F., Thomas-Vaslin V., Pena-Rossi C., Andrade L., SundBlad A., Forni L. et Coutinho A., «B cell activities in normal unmanipulated mice. », Contrib Microbiol Immunol, 11,1989, p. 1-26.

Gilbert S. F., SAPp J. ET TAuber A. I., «A symbiotic view of life: we have never been individuals. », Q Rev Biol, 87(4), 2012, p. 325-341.

Gottrand G., Courau T., Thomas-Vaslin V., Prevel N., Vazquez T., Ruocco M. G., Lambrecht B., Bellier B., Colombo B. M. et Klatzmann D., «Regulatory T cell development and function are impaired in mice lacking membrane expression of full length ICAM-1 », Immunology, 2015.

Hadrup S. R., Strindhall J., Kollgaard T., Seremet T., Johansson B., Pawelec G., THOR STRATEN P. ET WIKBY A., «Longitudinal studies of clonally expanded CD8 T cells reveal a repertoire shrinkage predicting mortality and an increased number of dysfunctional cytomegalovirus-specific T cells in the very elderly. » J Immunol, 176(4), 2006, p. $2645-2653$

Ilangumaran S., Ferbeyre G., «Editorial: Cytokines in inflammation, aging, cancer and obesity. » Cytokine, 2016.

INADERA H., «The immune system as a target for environmental chemicals: Xenoestrogens and other compounds. » Toxicol Lett, 164(3), 2006, p. 191-206.

IVANOV II., LITTMAN D.R., «Modulation of immune homeostasis by commensal bacteria »Curr Opin Microbiol, 14(1), 2011, p. 106-114.

IWASAKI A., MEDZHITOV R., «Control of adaptive immunity by the innate immune system », Nat Immunol, 16(4), 2015, p. 343-353.

Jacquemart F., Thomas-Vaslin V. Coutellec L., Pour une évaluation globale des OGM : Des perspectives épistémologiques renouvelées pour l'analyse des risques, 2016.

JERNE N. K., «Idiotypic networks and other preconceived ideas », Immunol reviews, vol. 79, 1984, p. 5-24.

JERNE N. K., «The generative grammar of the immune system », Science, vol. 229, 1985, p. 1057-1059.

Kinoshita M., TAKEDA K., «Microbial and dietary factors modulating intestinal regulatory T cell homeostasis », FEBS Lett, 588(22), 2014, p. 4182-4187. 
Klatzmann D., AbBas A. K., «The promise of low-dose interleukin-2 therapy for autoimmune and inflammatory diseases », Nat Rev Immunol, 15(5), 2015, p. 283-294.

Lemke H., Coutinho A., Opitz H.-G. et Gronowicz E. «Macrophages suppress direct Bcell activation by Lipopolysaccharide » Scand. J. Immunol, n 4, 1975, p. 707-719.

Ley R. E., Lozupone C. A., Hamady M., Knight R. et Gordon J. I. «Worlds within worlds: evolution of the vertebrate gut microbiota $»$ Nat Rev Microbiol, n 6(10), 2008, p. 776-788.

Loap P., Pascalie J., Bersini H. et Thomas-Vaslin V., (in preparation), «Cell-State Transition Tracking : Multi-Parameter Flow Cytometry and Modelling for Lymphocyte Population Dynamics Quantification».

McEwan C. H., Bersini H., Klatzmann D., Thomas-Vaslin V. et Six A., $A$ computational technique to scale mathematical models towards complex heterogeneous systems, COSMOS workshop ECAL 2011 Conference, Paris, Luniver Press.

MedZhitov R., «Pattern recognition theory and the launch of modern innate immunity » J Immunol, 191(9), 2013, p. 4473-4474.

Medzhitov R. et Janeway C., «<02 Medzhitow Janeway pattern self non self.pdf $>$, Science, vol. 296, 2002, p. 298-300.

Merleau-Ponty M., (1969). Prose du monde, Paris, Gallimard, 1969.

Nehar-Belaid D., Courau T., Derian N., Florez L., Ruocco M. G. et Klatzmann D.,«Regulatory T Cells Orchestrate Similar Immune Evasion of Fetuses and Tumors in Mice », J Immunol, 196(2), 2016, p. 678-690.

NeLSON J. L., «The otherness of self: microchimerism in health and disease » Trends Immunol, 33(8), 2012, p. 421-427.

Reiche E. M. V., Nunes S. O. V. et Morimoto H. K. «Stress, depression, the immune system, and cancer », The Lancet Oncology, 5(10), 2004, p. 617-625.

Rosen R., «A relational theory of biological systems », Bulletin of mathematicals biophysics, 20, 1958, p. 245-260.

Simpson R. J., Lowder T. W., Spielmann G., Bigley A. B., LaVoy E. C. et Kunz H., «Exercise and the aging immune system », Ageing Res Rev, 11(3), 2012, p. 404-420. 
Six A., Bellier B., Thomas-Vaslin V. et Klatzmann D., «Systems biology in vaccine design », Microb Biotechnol, 5(2), 2012, p. 295-304.

Six A., Mariotti-Ferrandiz M. E., Chaara W., Magadan S., Pham H. P., Lefranc M. P., Mora T., Thomas-Vaslin V., Walczak A. M. et Boudinot P. «The Past, Present, and Future of Immune Repertoire Biology - The Rise of Next-Generation Repertoire Analysis », Front Immunol, 4, 2013, p. 413.

Souza-e-Silva, H., W. Savino, R. A. Feijoo And A. T. Vasconcelos (2009). «A cellular automata-based mathematical model for thymocyte development »PLoS One 4(12): e8233.

STEINMAN L., «Elaborate interactions between the immune and nervous systems», Nat Immunol, 5(6), 2004, p.575-581.

STEWART J., The primordial VRM system and the evolution of vertebrate immunity,. Georgetown, Texas, R.G.Landes, 1994.

TAUBer A. I., «The immune system and its ecology » Philosophy of science, 75, 2008, p. 224-245.

Thomas-VASLin V., Influence de l'environnement cellulaire sur la dynamique et le répertoire des populations de lymphocytes B, Paris 6, Science de la vie, 1990.

THOMAS-VASLIN V., «A complex immunological idiotypic network for maintenance of tolerance », Front Immunol, 5: 369, (2014a).

Thomas-VAslin V., «Evaluation de la résilience et des perturbations des systèmes complexes», La lettre de l'inSHS du CNRS, Paris, CNRS, 2014b.

Thomas-VAsLin V., «Complexité multi-échelle du système immunitaire: Evolution, du chaos aux fractales», dans Glade N., et Stephanou A. (DIR.),. Le vivant critique et chaotique, Paris, Editions Matériologiques, $2015 \mathrm{~b}$.

ThOMAS-VASLIN V., «Understanding and modelling the complexity of the immune system», First Complex Systems Digital Campus World E-Conference 2015, Tempe, United States, Springer, 2015a.

THOMAS-VASLIN V., «Contribution à une évaluation globale des systèmes complexes et des perturbations: l'exemple du système immunitaire », 2016. (Texte en ligne: http://www.rechercheriskogm.fr/fr/page/evaglo - contributions). 
Thomas-Vaslin V., Altes H. K., De Boer R. J. et Klatzmann D. «Comprehensive assessment and mathematical modeling of $\mathrm{T}$ cell population dynamics and homeostasis» J Immunol, 180(4), 2008, p.2240-2250.

Thomas-Vaslin V., Andrade L., Freitas A. et Coutinho A. «Clonal persistence of B lymphocytes in normal mice is determined by variable region-dependent selection », Eur J Immunol, 21(9), 1991, p. 2239-2246.

Thomas-Vaslin V., Salaun J., Gajdos B., Le Douarin N., Coutinho A. et Bandeira A., «Thymic epithelium induces full tolerance to skin and heart but not to B lymphocyte grafts », Eur J Immunol, 25(2), 1995, p. 438-445.

Thomas-Vaslin V., Six A., Bellier B. et Klatzmann D., «Lymphocyte Dynamics and Repertoires, Biological Methods», dans Dubitzky W., Wolkenhauer O., Cho K.-H. et Yokota H., Encyclopedia of Systems Biology, New York, Springer, 2013a, p. 1145-1149.

Thomas-Vaslin V., Six A., Ganascia J. G. et Bersini H. «Dynamical and Mechanistic Reconstructive Approaches of $\mathrm{T}$ Lymphocyte Dynamics: Using Visual Modeling Languages to Bridge the Gap between Immunologists, Theoreticians, and Programmers », Front Immunol, 4: 300, 2013a.

Thomas-Vaslin V., Six A., Pham H. P., Dansokho C., ChaAra W., Gouritin B., BELLIER B. et KLATZMANN D. «Immunodepression \& Immunosuppression during aging». dans Immunosuppression. M. B. Portela. Brazil, InTech open acces publisher, 2012, p. 125-146.

Underhill P. A., et Kivisild T. «Use of y chromosome and mitochondrial DNA population structure in tracing human migrations », Annu Rev Genet, 41, 2007, p. 539564.

Vatanen T., Kostic A. D., D'Hennezel E., Siljander H., Franzosa E. A., Yassour M., Kolde R., Vlamakis H., Arthur T. D., Hamalainen A. M., Peet A., Tillmann V., Uibo R., Mokurov S., Dorshakova N., Ilonen J., Virtanen S. M., Szabo S. J., Porter J. A., Lahdesmaki H., Huttenhower C., Gevers D., Cullen T. W., KniP M.,. Group D. S et XAVIER R. J., «Variation in Microbiome LPS Immunogenicity Contributes to Autoimmunity in Humans », Cell, 165(4), 2016, p. 842-853. 
VibertJ., et Thomas-Vaslin V., «Modelling $\mathrm{T}$ Cell Proliferation: Dynamics Heterogeneity, Depending on Cell Differentiation, Age, and Genetic Background », PLos Comput Biol, 13 (3): e1005417.

WeLSH R. M.,«Private specificities of heterologous immunity », Curr Opin Immunol, 18(3), 2006, p. 331-337.

Yuan T., Jiao Y., de Jong S., Ophoff R. A., Beck S. et Teschendorff A. E.,«An integrative multi-scale analysis of the dynamic DNA methylation landscape in aging », PLoS Genet, 11(2): e1004996, 2015. 